\title{
X-ray diffraction measurements of plasticity in shock-compressed vanadium in the region of 10-70 GPa
}

J. M. Foster, A. J. Comley, G. S. Case, P. Avraam, S. D. Rothman, A. Higginbotham, E. K. R. Floyd, E. T. Gumbrell, J. J. D. Luis, D. McGonegle, N. T. Park, L. J. Peacock, C. P. Poulter, M. J. Suggit, and J. S. Wark

Citation: Journal of Applied Physics 122, 025117 (2017); doi: 10.1063/1.4994167

View online: https://doi.org/10.1063/1.4994167

View Table of Contents: http://aip.scitation.org/toc/jap/122/2

Published by the American Institute of Physics

\section{Articles you may be interested in}

The $\alpha-\gamma-\varepsilon$ triple point and phase boundaries of iron under shock compression

Journal of Applied Physics 122, 025901 (2017); 10.1063/1.4993581

The $\alpha-\omega$ phase transition in shock-loaded titanium

Journal of Applied Physics 122, 045902 (2017); 10.1063/1.4987146

Lagrangian technique to calculate window interface velocity from shock velocity measurements: Application for quartz windows

Journal of Applied Physics 122, 085901 (2017); 10.1063/1.4985788

Powder diffraction from solids in the terapascal regime

Review of Scientific Instruments 83, 113904 (2012); 10.1063/1.4766464

Loading-path dependent deformation of nanocrystalline Ta under single- and double-shock, and quasi-isentropic compression

Journal of Applied Physics 121, 115901 (2017); 10.1063/1.4978359

In-situ kinetics study on the growth of expanded austenite in AISI 316L stainless steels by XRD

Journal of Applied Physics 122, 025111 (2017); 10.1063/1.4993189

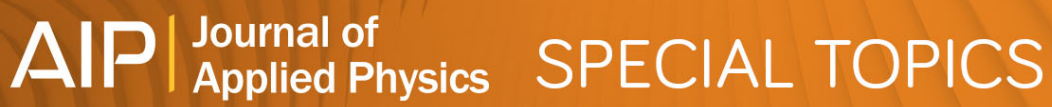

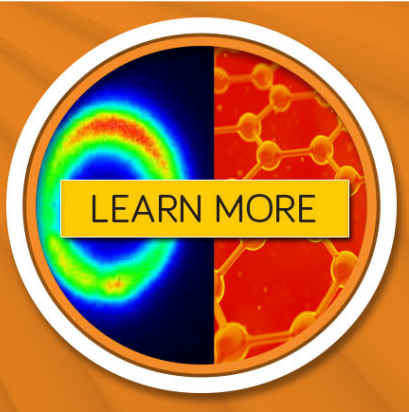




\title{
$\mathrm{X}$-ray diffraction measurements of plasticity in shock-compressed vanadium in the region of $10-70 \mathrm{GPa}$
}

\author{
J. M. Foster, ${ }^{1}$ A. J. Comley, ${ }^{1}$ G. S. Case, ${ }^{1}$ P. Avraam, ${ }^{1}$ S. D. Rothman, ${ }^{1}$ A. Higginbotham,${ }^{2}$ \\ E. K. R. Floyd, ${ }^{1}$ E. T. Gumbrell, ${ }^{1}$ J. J. D. Luis, ${ }^{1}$ D. McGonegle,${ }^{3}$ N. T. Park, ${ }^{1}$ L. J. Peacock, ${ }^{1}$ \\ C. P. Poulter, ${ }^{1}$ M. J. Suggit, ${ }^{3}$ and J. S. Wark ${ }^{3}$ \\ ${ }^{1}$ AWE Aldermaston, Reading RG7 4PR, United Kingdom \\ ${ }^{2}$ Department of Physics, York Plasma Institute, University of York, Heslington, York YO10 5DD, \\ United Kingdom \\ ${ }^{3}$ Department of Physics, Clarendon Laboratory, University of Oxford, Parks Road, Oxford OX1 3PU, \\ United Kingdom
}

(Received 11 April 2017; accepted 1 July 2017; published online 14 July 2017)

\begin{abstract}
We report experiments in which powder-diffraction data were recorded from polycrystalline vanadium foils, shock-compressed to pressures in the range of 10-70 GPa. Anisotropic strain in the compressed material is inferred from the asymmetry of Debye-Scherrer diffraction images and used to infer residual strain and yield strength (residual von Mises stress) of the vanadium sample material. We find residual anisotropic strain corresponding to yield strength in the range of 1.2 GPa-1.8 GPa for shock pressures below $30 \mathrm{GPa}$, but significantly less anisotropy of strain in the range of shock pressures above this. This is in contrast to our simulations of the experimental data using a multi-scale crystal plasticity strength model, where a significant yield strength persists up to the highest pressures we access in the experiment. Possible mechanisms that could contribute to the dynamic response of vanadium that we observe for shock pressures $\geq 30 \mathrm{GPa}$ are discussed.

[http://dx.doi.org/10.1063/1.4994167]
\end{abstract}

\section{INTRODUCTION}

The dynamic response of materials compressed at high strain rate by shock or ramp loading continues to attract significant experimental and theoretical interest. ${ }^{1-4}$ Shear stress in excess of a material's elastic limit results in plastic deformation by a number of candidate processes that include, among others, the atom-by-atom slip along lattice planes that is enabled by the creation and motion of dislocations, deformation twinning, and change of phase. These processes are strain and strain-rate dependent, and consequently a material's response may be significantly different from that found in quasi-static testing.

In the case of shock-wave loading, an initial elastic response is followed by plastic deformation if the yield stress [the Hugoniot elastic limit (HEL)] of the material is exceeded. Observation of the resulting elastic-plastic twowave structure at the macroscopic level (for example, by observing sequential elastic and plastic wavefronts via the time-dependent velocity at a free surface or interface) provides a means of investigating the integral result of processes at the invisible level of the crystal lattice. In contrast to such dynamic but macroscopic observations of plasticity, in situ measurements using $\mathrm{x}$-ray diffraction techniques enable a material's response at the detailed level of the crystal lattice to be directly probed. Dynamic, high-pressure experiments using $\mathrm{x}$-ray diffraction provide data complementary to quasistatic experiments in diamond-anvil cells ${ }^{5}$ that are also diagnosed by $\mathrm{x}$-ray techniques.

Significant progress has been made in x-ray diffraction-diagnosed, high-strain-rate experiments investigating the plastic response of the face-centred-cubic (fcc) metal copper $^{6}$ and the body-centred-cubic (bcc) metals iron and tantalum, ${ }^{7-11}$ and in molecular-dynamics (MD) modelling of these materials. ${ }^{8,12-14}$

There are little available experimental data on the highstrain-rate yield strength of vanadium. The limited number of publications that are available appears to show some inconsistencies, although they arise from experiments carried out under conditions of very different strain rates and length- and timescales and used material samples of possibly different initial microstructures. In shock-propagation experiments in vanadium in the pressure range of $2.9 \mathrm{GPa}-9.7 \mathrm{GPa}$, Chhabildas and Hills ${ }^{15}$ found a constant yield strength of $0.43 \mathrm{GPa}$. In ramp-driven experiments (at the Omega laser facility) investigating the stabilisation of Rayleigh-Taylor instability in vanadium in the pressure range of $77 \mathrm{GPa}-95 \mathrm{GPa}$, Park et al. ${ }^{16}$ reported an average yield strength of $2.5 \mathrm{GPa}$. In plate-impact experiments using a two-stage gas gun, Yu et al. ${ }^{17}$ found lower and upper limits for the yield strength between $0.3 \mathrm{GPa}$ and $2.0 \mathrm{GPa}$, for shock pressures in the range of $32 \mathrm{GPa}-88 \mathrm{GPa}$. They found an abrupt rise in the yield strength at around $60 \mathrm{GPa}$, possibly consistent with a change of phase from bcc to rhombohedral structure. Conversely, quasi-static diamondanvil-cell experiments by Klepeis et al. ${ }^{18}$ have indicated a yield strength increasing from $0.5 \mathrm{GPa}$ to $3.5 \mathrm{GPa}$ in the pressure range of $10 \mathrm{GPa}-50 \mathrm{GPa}$, followed by a reduction of strength in the pressure range of $50 \mathrm{GPa}-90 \mathrm{GPa}$ that they associate with the bcc-to-rhombohedral phase transition in vanadium. Diamond-anvil cell experiments by Jenei et al. ${ }^{19}$ confirm the existence of the bcc-to-rhombohedral phase transition at $61.5 \mathrm{GPa}$, but find that under non-hydrostatic conditions the phase transition occurs at $30 \mathrm{GPa}$ at ambient temperature and at $37 \mathrm{GPa}$ at $425 \mathrm{~K}$.

In this paper, we report time-resolved, in-situ x-ray diffraction data from samples of vanadium metal foils, 
compressed in planar loading by a shock wave launched from one surface. Our experiment is motivated by the requirement to investigate the high-strain-rate yield strength of vanadium and to test models used in dynamic simulations of material flow that incorporate plasticity.

The time-scale of our experiment is sufficiently small $(<10 \mathrm{~ns})$, and the loading sufficiently planar, that the macroscopic compression of the sample material remains uniaxial (there is insufficient time for a release wave to propagate inwards from the edges). Our aims are to observe the (initially uniaxial, subsequently plastic and non-uniaxial) response at the microscopic level of the crystal lattice; diagnose the strain state of the lattice following plastic flow from the observed distortion of Debye-Scherrer diffraction images; and (with an assumption about the shear-modulus of the shock-compressed vanadium) infer the yield strength of vanadium metal under the high strain-rate conditions of the experiment. Our x-ray diffraction images result from the volume-averaged strain within the $\mathrm{x}$-ray probe depth that we interrogate, and in detailed interpretation of our experiment, we use a 1-D hydrocode model incorporating a multi-scale crystal plasticity strength model for vanadium, described in Sec. V.

In brief summary, several continuum models for material strength exist. Of these, that due to Steinberg and Guinan ${ }^{20}$ does not depend on strain rate, whereas those due to Steinberg and Lund ${ }^{21}$ and Preston, Tonks, and Wallace ${ }^{22}$ include ratedependent effects and transition to phonon drag at the highest strain rates. The more-recent multiscale strength model due to Barton et $_{\text {al }}{ }^{23}$ includes a detailed treatment of the evolution of the dislocation density and dislocation velocity. A detailed comparison of the model of Barton et al. with experimental data for the elastic-to-plastic relaxation in shock-compressed single-crystal tantalum has been made by Wehrenberg et al. ${ }^{11}$ Continuum strength models such as those based on crystal plasticity theory have also been extended to incorporate account of dislocation density evolution and dislocation velocity, inherently include account of crystal anisotropy, and have been applied at high strain-rates. ${ }^{24,25}$ Such a model ${ }^{26}$ has been shown successfully to predict particle velocity profiles from both plate impact and laser shock experiments in singleand poly-crystal aluminium.

Higginbotham, Suggit et al., ${ }^{12}$ and Tramontina et al. ${ }^{13}$ have provided non-equilibrium molecular-dynamics (MD) simulations for single-crystal tantalum shock compressed along the [001] direction. The work by Tramontina et al. specifically include some pre-existing defects, which act as dislocation sources, and note the progressive importance of deformation twinning as a mechanism of plastic response, as the shock pressure increases: dislocations dominate at the lowest pressures, a combination of dislocations and twins is evident at $\sim 30 \mathrm{GPa}$, and twins dominate above $70 \mathrm{GPa}$. This succession of microstructures is said to agree well with the experimental data of $\mathrm{Lu}$ et al. ${ }^{28}$ and Florando et al..$^{29}$ Some notable success has also been obtained in the modelling of the particular case of the bcc metal iron in the single-crystal form, shocked along the [001] direction. ${ }^{30}$ There are few MD treatments of the response of the bcc metal vanadium to high-strain-rate compression.
The remaining sections of this paper are organised as follows. Section II provides details of our experimental setup, and Sec. III provides details of the texture of the polycrystalline vanadium-foil sample that we have used as well as our treatment of strain anisotropy in the analysis of the $\mathrm{x}$ ray diffraction data. Sections IV and V discuss the analysis of the x-ray diffraction data and its accuracy, and modelling of the experiment using a 1-D hydrocode model incorporating a multi-scale crystal plasticity strength model. Finally in Sec. VI, we discuss the possible importance of deformation twinning, phase change, and homogeneous nucleation of defects at pressures above $\sim 30 \mathrm{GPa}$ as an explanation for our $\mathrm{x}$-ray diffraction data.

\section{EXPERIMENTAL DETAILS}

The experiments were carried out at the Orion laser facility at AWE, Aldermaston. ${ }^{31}$ The experimental arrangement is shown schematically in Figs. 1 and 2. It uses a modified version of the X-ray diffraction camera (known as "BBXRD") described by Comley et al. ${ }^{10}$ and Higginbotham et al., ${ }^{32}$ and in the present experiment, a monochromatic x-ray source is used (Comley and Higginbotham used a spectrally broadband x-ray source). Five beams of Orion are used ablatively to drive a shock into a polycrystalline vanadium-foil laser target that is mounted in the plane of the square base of the pyramidshaped BBXRD enclosure that contains X-ray imaging plates at its four sides, where X-ray diffraction data are recorded. A further Orion laser beam illuminates a separate, pinholeapertured target that provides a source of near-monochromatic $\mathrm{X}$-ray line-radiation for diffraction from the sample material. This X-ray probe enters through one face of BBXRD where it is collimated and illuminates the vanadium-foil sample when

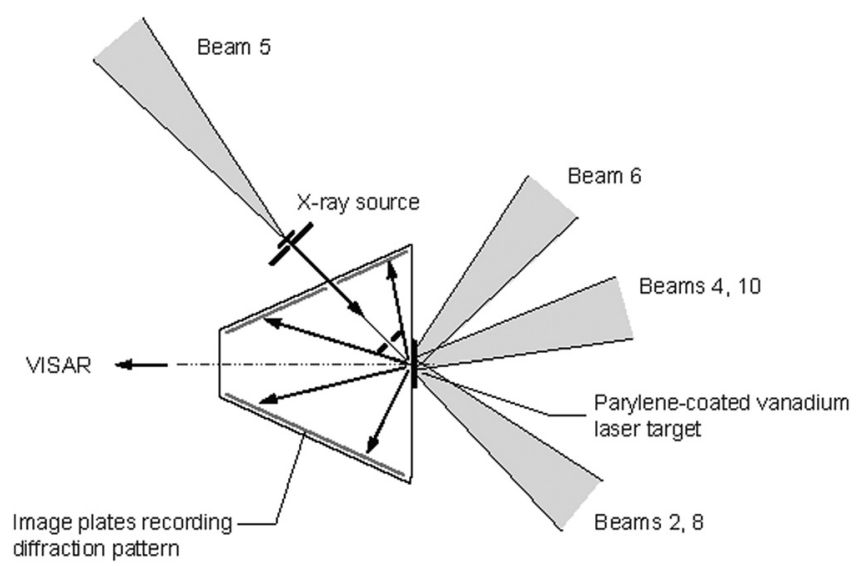

FIG. 1. Schematic of the experimental arrangement. One face of a vanadium-foil sample is coated with a Parylene ablator that is illuminated by five beams of the Orion laser. Laser ablation provides the pressure source that drives a near-planar shock through the sample. A pinhole-apertured $\mathrm{x}$ ray source illuminated by another beam of Orion (delayed in time) provides a collimated source of radiation that is incident on the sample whose uniaxial compression and subsequent plastic response are diagnosed by x-ray diffraction. The diffraction pattern is recorded on image-plate detectors, situated at the four sides of a pyramidal camera enclosure (BBXRD) that surrounds the rear surface of the sample. Shock breakout from the rear (undriven) surface of the vanadium foil is recorded using VISAR. A time-gated $\mathrm{X}$-ray camera is used to record $\mathrm{x}$-ray emission from the laser-ablated surface as a diagnostic of the uniformity of the incident laser intensity. 


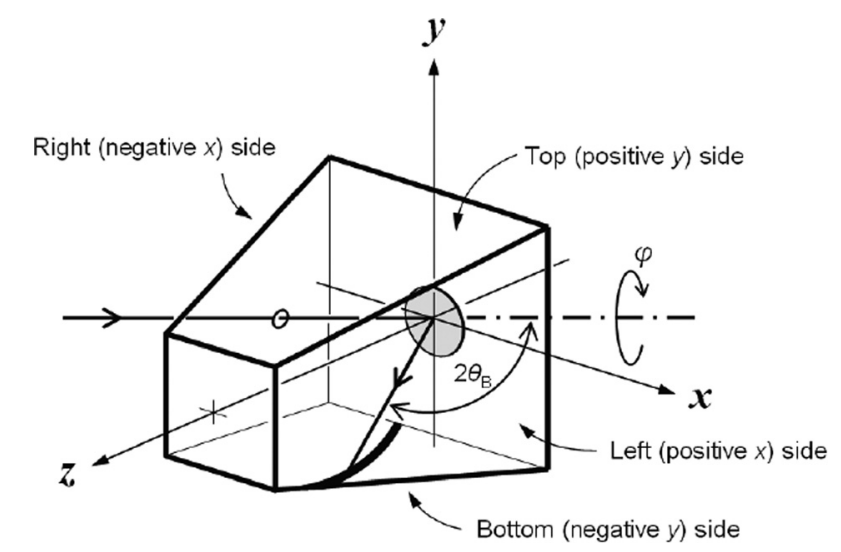

FIG. 2. Coordinate system for analysis of the diffraction images from a polycrystalline vanadium sample situated as shown in the $x, y$ plane at the base of the pyramidal BBXRD x-ray diffraction camera. The ray path from the collimated $\mathrm{x}$-ray source lies in the $x, z$ plane and is incident at $45^{\circ}$ at the surface of the sample. Debye-Scherrer diffraction from (002) planes of vanadium is shown schematically on the bottom (negative- $y$ side) image platecompare Fig. 4(b).

the shock wave has progressed approximately half way through its thickness. In this way, Debye-Scherrer diffraction images from both the undisturbed material ahead of the shock and the shock-compressed vanadium are recorded simultaneously. The $45^{\circ}$ angle of incidence of the $\mathrm{x}$-ray probe beam relative to the direction of propagation of the shock was chosen to provide sensitivity in the diffraction images to anisotropic strain of the shock-compressed vanadium.

Specific details are as follows. The vanadium-sample laser targets each consisted of a commercially available ${ }^{33}$ rolled vanadium foil of $10 \mu \mathrm{m}$ thickness and $99.8 \%$ purity, coated on one face with a $20-\mu \mathrm{m}$ Parylene-N ablator layer and a further flash coating of aluminium of $200 \mathrm{~nm}$ thickness. This foil sample was mounted over the 3-mm diameter hole in a tungsten-alloy ("Heavimet") washer, itself mounted from the square base of BBXRD. The laser target for the $\mathrm{x}$ ray probe was a vanadium foil of $5-\mu \mathrm{m}$ thickness mounted directly over the $0.5-\mathrm{mm}$ diameter hole of a $5-\mathrm{mm}$ square (outside dimensions) tantalum-foil pinhole. The five laser drive beams originated from the even-number-beam cluster of the Orion laser. The laser pulse shape was of $5 \mathrm{~ns}$ full width at half maximum (FWHM) duration, with $<200$ ps rise and fall times, and a plateau region with typically $\pm 10 \%$ temporal variations from constant power over the duration of the pulse. The drive laser beams were defocused to provide 5-mm diameter overlapping laser spots at the surface of the vanadium target. This defocus and overlapping of laser spots provided adequate spatial uniformity of intensity, without the use of phase plates. Total (all five beams) laser energy was in the range of 100-700 J, resulting in the incident laser intensity in the range approx. $6 \times 10^{10}-5 \times 10^{11} \mathrm{~W} \mathrm{~cm}^{-2}$ (and pressure in the vanadium in the range approx. 10-70 GPa). The vanadium target for the $\mathrm{x}$-ray probe was illuminated by one beam (0.5-ns duration "square" pulse, $0.35-\mu \mathrm{m}$ wavelength) from the Orion odd-number-beam cluster, at nearnormal incidence and defocused to $300-\mu \mathrm{m}$ spot size (approx. $1 \times 10^{15} \mathrm{~W} \mathrm{~cm}^{-2}$ incident intensity). The separation between this target and the vanadium foil sample was $45 \mathrm{~mm}$, and radiation from the $\mathrm{x}$-ray source was collimated, within the body of BBXRD, by a $600-\mu$ m diameter tantalum pinhole situated $10 \mathrm{~mm}$ from the vanadium sample. This arrangement resulted in illumination by the X-ray probe beam of a spot of approx. 800- $\mu \mathrm{m}$ diameter at the centre of the vanadium sample, and the angular collimation of the X-ray probe at the sample was better than $0.5^{\circ}$ FWHM. BBXRD was manufactured from a single block of stainless steel, machined to provide a $60-\mathrm{mm}$ square base and sides sloping at an angle of a $24.4^{\circ}$ relative to its axis. Imaging plates are located directly in contact with its four sloping sides and are maintained in place by small rare-earth magnets located in recesses within the sides. The BBXRD diagnostic was carefully characterised ${ }^{34}$ by using a coordinate measuring machine (CMM), to determine the flatness, angle, and position (perpendicular distance, relative to the centre of the sample) of each image plane with $10-\mu \mathrm{m}$ accuracy. BBXRD is shielded by tantalum plates on the outer surface of each of its four sides.

The x-ray source used to record the Debye-Scherrer diffraction images originated predominantly from the line radiation of helium-like vanadium $\left(2{ }^{1} \mathrm{P}-1{ }^{1} \mathrm{~S}: \lambda=2.3817 \AA\right.$; $2{ }^{3} \mathrm{P}-1{ }^{1} \mathrm{~S}: \lambda=2.3931 \AA$ ) together with its lithium-like satellite lines. The overall spectral width was approx. $0.02 \AA$ FWHM. The use of a relatively long-wavelength $\mathrm{x}$-ray probe was necessitated by the need to work below the $\mathrm{K}$ absorption edge $(5.465 \mathrm{keV})$ of vanadium, where the sample foil was sufficiently transparent (20- $\mu \mathrm{m}$ mean free path) to the x-ray probe. In consequence, reflection from only three crystallographic planes was possible: $(011), 2 \theta_{\mathrm{B}}=67.71^{\circ}$ for the ambient material; (002), $2 \theta_{\mathrm{B}}=103.97^{\circ}$ for the ambient material; and (112), $2 \theta_{\mathrm{B}}=149.56^{\circ}$ for the ambient material. Of these, only the (011) and (002) planes provided possible reflections for the shock-compressed material. Of course, a shorter-wavelength $\mathrm{x}$-ray probe would provide access to a greater number of reflecting planes, but a probe mean free path of $\geq 20 \mu \mathrm{m}$ would require a line-radiation $\mathrm{x}$-ray source of $\geq 10 \mathrm{keV}$ : a sufficiently bright such source could not be excited with one Orion long-pulse laser beam in the current experimental set-up, but should be accessible in future work by using one of the two Orion petawatt beams.

Fuji BAS Type SR imaging plates were used to record the $\mathrm{X}$-ray diffraction images and were scanned with $50-\mu \mathrm{m}$ spatial resolution. We note that although the sensitive phosphor layer of the SR-type image plate has a thickness of $120 \mu \mathrm{m}$, the mean free path of the $5-\mathrm{keV}$-ray probe in the phosphor is approx. $15 \mu \mathrm{m}$ : the image plate therefore acts essentially as a planar detector. A thin vanadium foil filter was placed adjacent to each image plate, to reduce $\mathrm{x}$-ray background signal arising from the laser-illuminated main target.

A time delay of typically $5 \mathrm{~ns}$ between the laser beams driving the shock and the $\mathrm{x}$-ray source allowed for the shock's transit time through the Parylene ablator and partly through the vanadium sample and enabled diffraction images from both the undisturbed and the shock-compressed vanadium to be recorded simultaneously.

In addition to BBXRD, two other target diagnostics were employed.

A two-channel VISAR (Velocity Interferometer System for Any Reflector) was used to record the time of shock 
breakout as well as the free-surface velocity of the vanadium sample. This system uses a probe laser of $532 \mathrm{~nm}$ wavelength and $50 \mathrm{~ns}$ pulse duration, and line imaging data were recorded within a $25 \mathrm{~ns}$ window, on two streak cameras, from interferometer beds viewing the same region of the vanadium laser target. Etalons of 7 and $10 \mathrm{~mm}$ thickness provided velocity per fringe sensitivities of 7.118 and $4.983 \mu \mathrm{m} \mathrm{ns}^{-1}$. Temporal resolution was approximately 100 ps. Fringe phase (and hence free-surface velocity) was extracted from the VISAR interferometer data by the Fourier-transform method described by Celliers et al. ${ }^{35}$ The effective spatial resolution of VISAR data is determined by the width of the bandpass spatial-frequency filter used in the Fourier-transform data reduction, and in our present experiment, we estimate a spatial resolution of approximately $100 \mu \mathrm{m}$. Celliers et al. ${ }^{35}$ note that the determination of fringe phase is subject to systematic uncertainty in a VISAR system of our type and is typically around \pm 0.05 fringe. We therefore anticipate a systematic uncertainty of free-surface velocity of order $\pm 0.3 \mu \mathrm{m} \mathrm{ns}^{-1}$, and this is typically evident in our measurements (as we discuss in Sec. IV). VISAR viewed the surface of the vanadium target through a hole in the apex of BBXRD and imaged along a diameter of the driven region of the target.

A time-gated X-ray pinhole camera was used to record $\mathrm{X}$-ray emission from the laser-illuminated surface of the target.

\section{TEXTURE OF THE VANADIUM SAMPLE AND SENSITIVITY TO ANISOTROPY OF STRAIN}

The rolled vanadium foil used for this experiment was found to have a significant metallurgical texture, resulting in incomplete Debye-Scherrer diffraction rings. Electron backscatter diffraction (EBSD) measurements ${ }^{36}$ showed grains of size 3-5 $\mu \mathrm{m}$, and EBSD pole figures (discussed below) indicate that these grains are oriented primarily with the [001] direction normal to the surface of the sample.

For incident (x-ray probe) wave vector $\mathbf{k}_{0}$ and a particular choice of direction for diffracted wave vector $\mathbf{k}$, the condition $\mathbf{k}=\mathbf{k}_{0}+\mathbf{G}$ for $\mathrm{x}$-ray diffraction requires the existence within the sample of grains with appropriately oriented reciprocallattice vector $\mathbf{G}$. This, together with the requirement to obtain maximum sensitivity to anisotropic strain in the experiment, determined our choice of orientation of the vanadium foil (angular orientation around the axis of shock propagation) when it was mounted on BBXRD for the experiment.

Higginbotham and McGonegle ${ }^{37}$ have considered in detail the case of Debye-Scherrer diffraction from arbitrarily strained materials and we follow their treatment here. If the included angle between the direction of shock propagation $\mathbf{n}$ (the positive- $z$ direction of Fig. 2) and $\mathbf{G}$ is $\psi$, and with the simplifying assumptions that the shock and transverse directions are principal axes of strain and that strain is continuous throughout the sample (Voigt condition), then sensitivity to anisotropic strain arises from the relation ${ }^{37}$

$$
\left(\frac{d}{d_{0}}\right)^{2}=\left(1+\varepsilon_{T}\right)^{2}+\left[\left(1+\varepsilon_{S}\right)^{2}-\left(1+\varepsilon_{T}\right)^{2}\right] \cos ^{2} \psi .
$$

Here, $d_{0}$ and $d$ are the lattice-plane spacings (obtained from their respective Bragg angles, $\theta_{\mathrm{B}}$ ) of the undisturbed and strained material, and $\varepsilon_{S}, \varepsilon_{T}$ are elastic lattice strains in the shock and transverse directions. Clearly, sensitivity of the experiment to strain anisotropy requires that we record a significant variation of $\psi$ within the azimuthal region of the Debye-Scherrer ring accessible to measurement.

With reference to Fig. 2, we define the direction of the diffracted wave vector $\mathbf{k}$ by its polar angle $2 \theta_{\mathrm{B}}$ relative to the direction of the incident (x-ray probe) wave vector $\mathbf{k}_{0}$, and by azimuthal angle $\varphi$. We define $\varphi=0$ as lying in the $x, z$ plane. We deliberately oriented the vanadium sample so that for the (002) reflection, the most intense region of the Debye-Scherrer diffraction ring was recorded on the "bottom" (negative- $y$ side) image-plate of BBXRD, within the range of azimuthal angles $-90^{\circ} \leq \varphi \leq-30^{\circ}$ corresponding to an adequately large range of $\psi\left(0.5<\cos ^{2}(\psi)<0.9\right)$. This was achieved by mounting the vanadium foil (in the $x, y$ plane) with its rolling direction at an angle of approximately $45^{\circ}$ to the $y$ axis. The texture of the rolled vanadium foil that we used was sufficiently well oriented that under these conditions, the measurable intensity for the (002) reflection was present on only part (see Fig. 4) of the "left" (positive- $x$ side) image plate, and no measureable intensity was recorded on the "top" (positive- $y$ side) image-plate. The (011) reflection was recorded on the left-side image plate only, but this is because the geometry of BBXRD does not permit recording its (smaller) Bragg angle on the other image plates.

Although EBSD pole figures do not represent the complete orientation distribution function (ODF) of the sample, ${ }^{38}$ it is nevertheless interesting to consider them for the rolled vanadium foil that we used, as they provide some information on the initial conditions of the experiment. Figures 3(a) and 3(b) show our measured pole figures for the [002] and [011] directions of grains within the vanadium foil. We show each pole figure in equal-area projection, oriented as in the experiment and in the coordinate system of Fig. 2, and viewed as if from inside BBXRD. For the He-like resonance line of vana$\operatorname{dium}(\lambda=2.3817 \AA)$ used as the $\mathrm{x}$-ray probe in these experiments, the red overlay in each pole figure indicates the positions in the ODF of reciprocal-lattice vector points necessary to complete the entire Debye-Scherrer ring. Similarly, the grid shown (in white) as an overlay is labelled according to the $2 \theta_{\mathrm{B}}\left(0^{\circ}, 30^{\circ}, \ldots 120^{\circ}\right.$ indicated $)$ and $\varphi\left(-60^{\circ},-30^{\circ}, \ldots 60^{\circ}\right.$ indicated) directions of diffracted wave vector $\mathbf{k}$ that would result from a reciprocal-lattice vector occupying the positions indicated in the ODF. As is clear from Fig. 3(a), the texture is sufficiently well oriented that for the (002) reflection, only the region $-60^{\circ} \leq \varphi \leq 0^{\circ}$ (as found approximately in the experiment) contributes significant intensity to the diffraction image; there are very few grains oriented to provide diffracted intensity in the other directions potentially recorded by BBXRD. Figure 3(b) indicates that for the (011) reflection, greatest intensity in the diffraction images occurs only near the regions $-60^{\circ} \leq \varphi \leq-30^{\circ}$ and $30^{\circ} \leq \varphi \leq 60^{\circ}$ (again, approximately as observed in the experiment).

Since vanadium is known to be susceptible to deformation twinning, ${ }^{39}$ and since for our textured sample the plane of maximum resolved shear stress is close to the (112) twin composition planes for grain orientations with a high probability of occurrence, it is interesting to consider where 

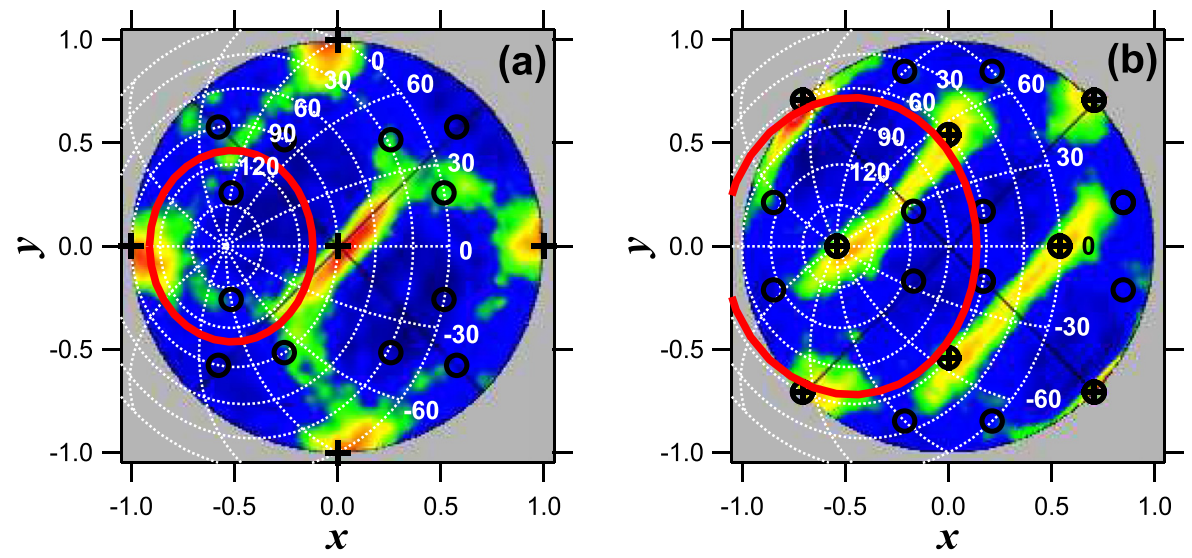

FIG. 3. Electron backscatter-diffraction pole figures showing the orientation of the [002] and [011] directions [(a) and (b), respectively] in the rolled vanadium-foil sample. The red lines indicate the trajectories of reciprocal-lattice vector points in each pole figure that potentially contribute to the observed reflections in the experiment $\left(2 \theta_{\mathrm{B}}=104^{\circ}\right.$ and $\left.2 \theta_{\mathrm{B}}=68^{\circ}\right)$, for an incident probe wavelength of $2.3817 \AA$. For arbitrary incident wavelength, $2 \theta_{\mathrm{B}}$ and $\varphi$ in the resulting diffraction pattern are indicated by the grid lines shown in white. The points shown in black $(+, o)$ are the directions of an imaginary, well-aligned grain before (+) and after (o) twinning on all possible (112) planes. Each pole figure is shown in equal-area projection, oriented in the coordinate system of Fig. 2, and viewed as if from the inside of the BBXRD camera. The colour scale is logarithmic.

evidence for deformation twins in diffraction images from the compressed material might appear. We therefore show two further items of information in Figs. 3(a) and 3(b). The points represented by single crosses $(+)$ indicate the positions within the pole figure of an idealised single grain of the ambient material, oriented as shown. The points shown by open circles (o) show the directions within each pole figure resulting from all possible twin faults on (112) planes of this idealised single grain.

\section{X-RAY DIFFRACTION DATA}

Figure 4 shows representative $\mathrm{x}$-ray diffraction images from the left (positive- $x$ side) and bottom (negative- $y$ side) image plates of BBXRD. Reflections from the (002) planes of the ambient and shocked vanadium occur close to $2 \theta_{\mathrm{B}}=104^{\circ}$ and $110^{\circ}$, respectively, and are evident in both images. Their extent in the azimuthal $(\varphi)$ direction is consistent with the pre-shot EBSD pole-figure characterisation of the vanadium foil, as explained in Sec. III. Similarly, the left-side image plate shows the (011) reflections from the ambient and shocked vanadium, close to $2 \theta_{\mathrm{B}}=68^{\circ}$ and $72^{\circ}$. The (112) reflection from the ambient material is just captured at the extreme edge of the bottom image plate, at
$2 \theta_{\mathrm{B}}=150^{\circ}$. The data of Fig. 4 (Shot 5352 of Table I) were obtained at an incident laser intensity of $1.7 \times 10^{11} \mathrm{~W} \mathrm{~cm}^{-2}$, and the inferred volumetric compression $\left(V_{0} / V=1.13\right)$ implies a peak sample pressure (mean stress) of $23 \mathrm{GPa}$ (as discussed in more detail below).

By changing the laser energy at constant laser spot size over a number of experimental shots, we have obtained similar x-ray diffraction images at approximately equally spaced points within the pressure range of 10-70 GPa. We summarise this body of data in Figs. 5 and 6, which show representative examples (Fig. 5) and our complete data set (Fig. 6) from several such measurements, obtained over three experimental runs. Figure 5 shows intensity distributions ("lineouts") along several lines of constant azimuthal angle (positions around the Debye-Scherrer ring) for the (002) reflection, obtained from the bottom image plate of BBXRD; Fig. 6 summarises the observed reflection-peak positions. Consistently, we find that for the compressed material, there is a dependence-presumably arising from anisotropy of strain —of the observed position of the (002) reflection on azimuthal position on the Debye-Scherrer ring, up to a pressure of approx. $30 \mathrm{GPa}$. Above that pressure, we find no unambiguous (within the accuracy and reproducibility of the experiment) dependence of peak position on the azimuthal angle-although at the
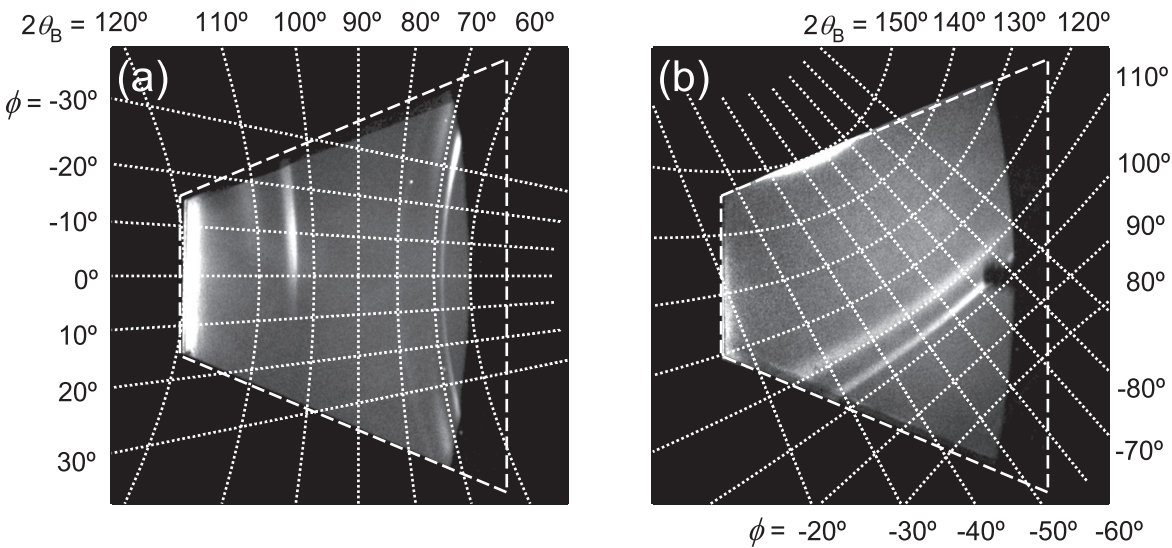

FIG. 4. Raw data from (a) the left (positive- $x$ side) and (b) bottom (negative- $y$ side) image plates of BBXRD. The (011) reflections of the ambient and compressed vanadium occur close to $2 \theta_{\mathrm{B}}=68^{\circ}$ and $72^{\circ}$. Reflections from (002) planes occur close to $2 \theta_{\mathrm{B}}=104^{\circ}$ and $110^{\circ}$. Azimuthal positions around the Debye-Scherrer ring are shown by the $\varphi$ coordinate. Both images are oriented as if viewed from inside BBXRD. 
TABLE I. Quantities inferred from the x-ray-diffraction and VISAR data for all experimental shots reported in Fig. 6. The uncertainty of measurement of strain is \pm 0.002 under the most favourable conditions; data in square brackets have sufficiently greater uncertainty that the corresponding shear strain and inferred von Mises stress cannot be unambiguously identified and so are not reported (see text). Uncertainty in the pressure inferred from VISAR represents the range of free-surface velocity recorded over the full 2-mm diameter of the sample material.

\begin{tabular}{|c|c|c|c|c|c|c|c|c|}
\hline Shot No. & $\varepsilon_{\mathrm{T}}( \pm 0.002$ or greater $)$ & $\varepsilon_{S}( \pm 0.002$ or greater $)$ & $V_{0} / V$ & $T(\mathrm{~K})$ & $P$ from strain $(\mathrm{GPa})$ & $P$ from VISAR $(\mathrm{GPa})$ & $G(\mathrm{GPa})$ & $\bar{\sigma}(\mathrm{GPa})$ \\
\hline 5357 & -0.019 & -0.030 & 1.072 & 340 & 11.7 & $14.5 \pm 3$ & 53.2 & $1.2 \pm 0.3$ \\
\hline 3755 & -0.024 & -0.036 & 1.088 & 350 & 14.7 & $20.3 \pm 4$ & 54.5 & $1.4 \pm 0.3$ \\
\hline 3739 & -0.026 & -0.038 & 1.095 & 360 & 16.1 & $18.3 \pm 4$ & 55.0 & $1.3 \pm 0.3$ \\
\hline 4392 & -0.027 & -0.043 & 1.103 & 370 & 17.6 & $16.8 \pm 4$ & 55.6 & $1.8 \pm 0.3$ \\
\hline 5352 & -0.036 & -0.047 & 1.130 & 410 & 23.0 & $22.7 \pm 3$ & 57.6 & $1.2 \pm 0.3$ \\
\hline 5055 & -0.051 & -0.050 & 1.169 & 480 & 31.6 & $\ldots$ & 60.6 & $-0.2 \pm 0.3$ \\
\hline 4393 & -0.063 & -0.058 & 1.209 & 610 & 41.5 & $35.3 \pm 4$ & 63.6 & $-0.6 \pm 0.4$ \\
\hline 5345 & -0.063 & -0.064 & 1.216 & 640 & 43.3 & $39.7 \pm 7$ & 64.0 & $0.2 \pm 0.4$ \\
\hline 5347 & -0.068 & -0.070 & 1.239 & 720 & 49.0 & $49.2 \pm 12$ & 65.6 & $0.2 \pm 0.4$ \\
\hline 4401 & {$[-0.085]$} & {$[-0.075]$} & 1.291 & 990 & 64.4 & $67.8 \pm 5$ & 69.1 & $\ldots$ \\
\hline 5349 & {$[-0.081]$} & {$[-0.080]$} & 1.287 & 960 & 63.0 & $60.0 \pm 16$ & 68.8 & $\ldots$ \\
\hline 5348 & {$[-0.084]$} & {$[-0.081]$} & 1.296 & 1030 & 66.1 & $76.1 \pm 17$ & 69.4 & $\ldots$ \\
\hline 5350 & {$[-0.085]$} & {$[-0.082]$} & 1.302 & 1070 & 68.1 & $81.9 \pm 13$ & 69.8 & $\ldots$ \\
\hline 4408 & {$[-0.090]$} & {$[-0.085]$} & 1.321 & 1210 & 74.4 & $76.1 \pm 5$ & 70.8 & $\ldots$ \\
\hline
\end{tabular}

highest pressures $(50-70 \mathrm{GPa})$ that we have accessed, the increased width of the diffraction line and the increased diffuse background signal on the BBXRD image plates make accurate determination of the position of line centre difficult.

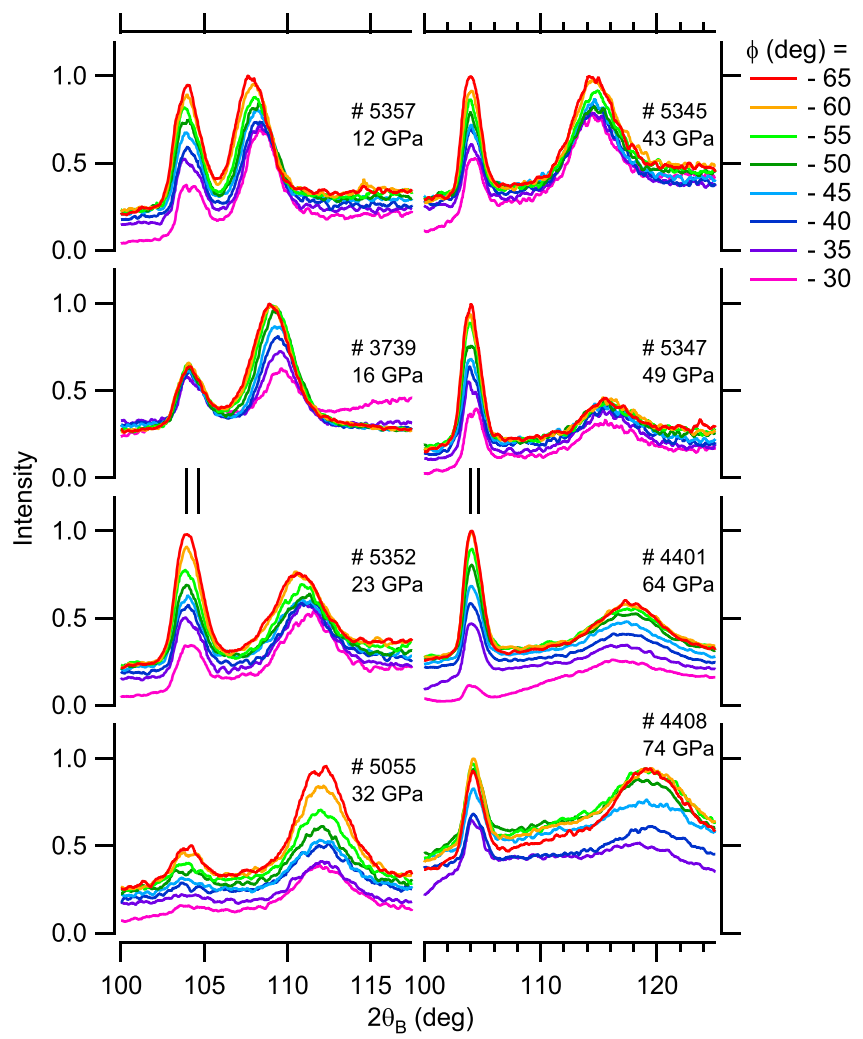

FIG. 5. Intensity distribution for the (002) reflection from ambient and shock-compressed vanadium, at different angular positions $(\varphi)$ around the Debye-Scherrer ring, and for representative experiments identified individually by shot number. The tick marks represent the expected position of reflections from the ambient material, for probe wavelengths of $2.3817 \AA$ and $2.3931 \AA$ (resonance and inter-combination lines of helium-like vanadium), and provide an indication of the contribution of the spectral width of the $\mathrm{x}$-ray source to the angular resolution of the experiment. The data are labelled by shot number and by pressure (rounded to two figures) inferred from the measured strain (see also Table I).
The (011) reflection covers a range of azimuthal angle that is too small to provide a measurement of strain anisotropy, but its position is nevertheless consistent with the (002) data.

We characterise the position and width of the diffraction line by a Gaussian fit to the experimental line profile. Figure 7 summarises the observed full width at half maximum intensity (FWHM) angular width of the (002) reflection,

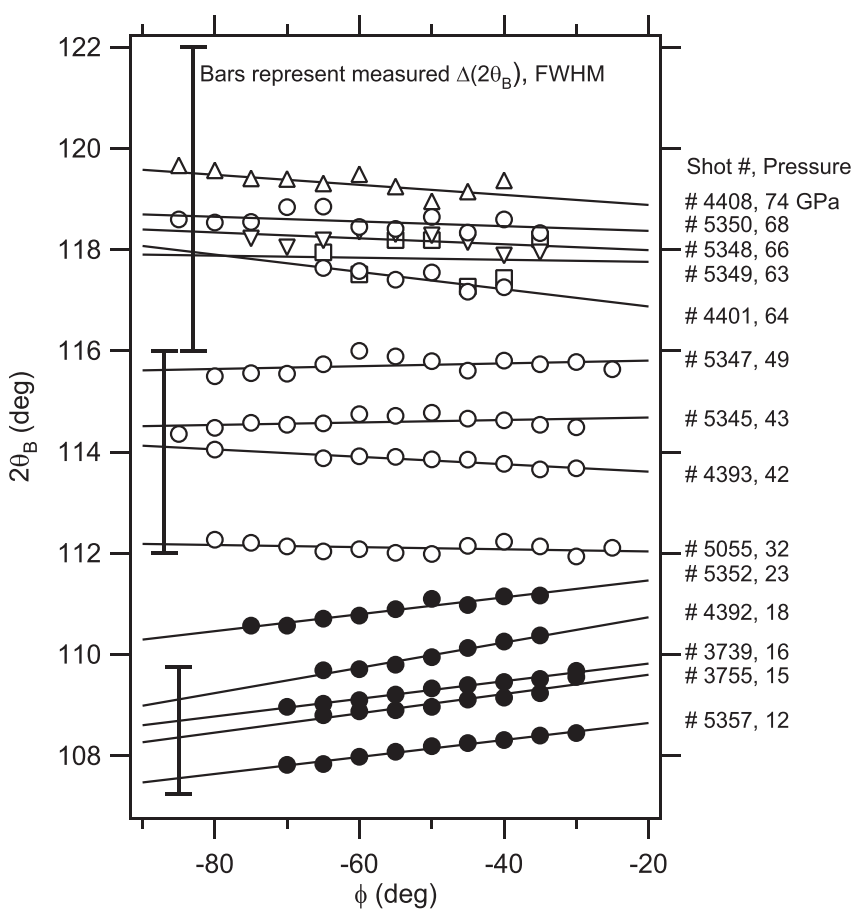

FIG. 6. Angles ( $\left.2 \theta_{\mathrm{B}}\right)$ of peak reflection for the shock-compressed vanadium, as a function of angular position $(\varphi)$ around the Debye-Scherrer ring, for the full set of experimental shots and with drive pressures in the range of 12-74 GPa (see Table I). The lines through the data points are simply a guide to assess the scatter of the data; they have no other significance. Filled and open data points simply discriminate the unambiguous presence or not of detectable anisotropy of strain. The data are labelled by shot number and by pressure (rounded to two figures) inferred from the measured strain (see also Table I). 


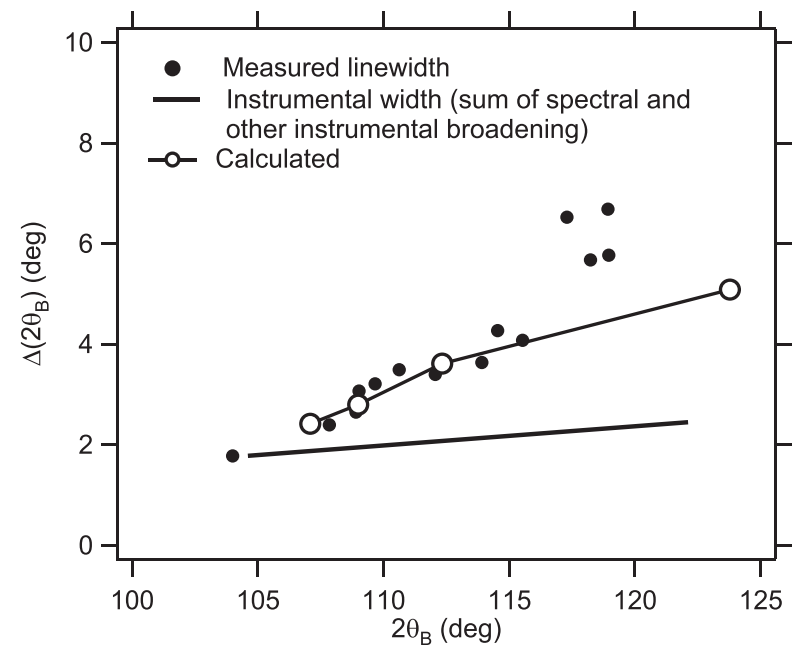

FIG. 7. Angular width, $\Delta\left(2 \theta_{\mathrm{B}}\right)$, of the (002) reflection from the shockcompressed vanadium, measured at $\varphi=-65^{\circ}$. The solid black line indicates the instrumental width. The open-circle data points are from calculations of the expected diffraction line width (see text, Sec. V).

as a function of peak position (compression of the vanadium sample). The indicated instrumental width arises from the spectral width of the source and all other contributions to the instrument function (dominated by the finite angular collimation of the source) and was assessed from the measured FWHM width of the reflection from the ambient material. It is non-constant because of the increase of spectral dispersion with an increase of Bragg angle.

The variation of reflection-peak position with the azimuthal angle shown in Fig. 6 is small $\left(\leq 2^{\circ}\right.$ variation of peak position over the range for $-90^{\circ} \leq \varphi \leq 0^{\circ}$ ), and we have therefore been concerned to demonstrate that this is not an artefact of, for example, departure from flatness of the image plates, errors of image plate registration, or other departures of the assumed dimensions of BBXRD from those obtained from the CMM metrology. We have made an estimate of systematic errors in our measurement of the Bragg-reflection position by using a static copper-foil sample in conjunction with a helium-like iron $\left(2^{1} \mathrm{P}-1{ }^{1} \mathrm{~S}: \lambda=1.8503 \AA{ }^{3} ; 2^{3} \mathrm{P}-1{ }^{1} \mathrm{~S}\right.$ : $\lambda=1.8591 \AA$ ) $\mathrm{x}$-ray source. A greater number of reflections are recorded from the copper sample than from vanadium (because of the smaller wavelength of the x-ray probe), and furthermore the helium-like resonance and inter-combination lines of iron are partially resolved in the diffraction images. We used a sample of copper foil with a little evident texture, so that data were recorded on all four image plates of BBXRD. Figure 8 summarises the positions of the (222), (113), and (022) reflections from copper, presented in a way to facilitate direct comparison with Fig. 6. Over the range of Bragg angles $92^{\circ} \leq 2 \theta_{\mathrm{B}} \leq 126^{\circ}$ (a greater range than covered for the vanadium sample), we find systematic and random errors of peak position $\left(2 \theta_{\mathrm{B}}\right)$ of typically $0.2^{\circ}$.

Our treatment of the vanadium data makes the simplifying assumption that the deformation of all crystallites within the sample, whatever their initial orientation, arises from a single deformation gradient (Voigt condition) and it follows the method outlined by Higginbotham and McGonegle. ${ }^{37}$ In the context of Eq. (1), we infer lattice-plane spacing $\left(d / d_{0}\right)$

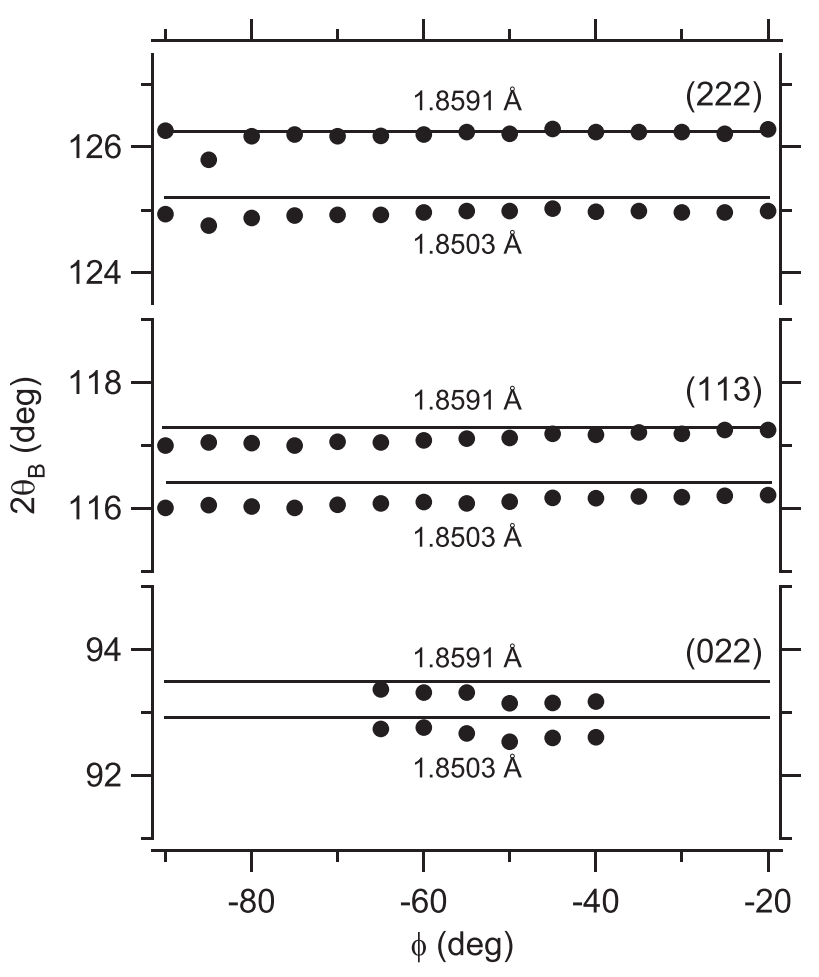

FIG. 8. Angles $\left(2 \theta_{\mathrm{B}}\right)$ of peak reflection from copper, for a helium-like iron $\mathrm{x}$-ray probe (primarily, $\lambda=1.8503 \AA$ and $\lambda=1.8591 \AA$ ) and for the reflection planes indicated. The data points are the experimental measurements; the lines show the expected positions for the nominal lattice constant. The figure is presented in a form for a direct comparison with Fig. 6 .

from the measured diffraction angle $\left(\theta_{\mathrm{B}}\right)$ and angle $\psi$ from the known geometry of the experiment. Figure 9 summarises linear fits of these data to Eq. (1), for those cases where in our judgement the experimental data are unambiguous. The error bars shown in Fig. 9 represent uncertainties under the most favourable conditions: that is by assuming systematic and random errors of diffraction peak position $\left(2 \theta_{\mathrm{B}}\right)$ of typically $0.2^{\circ}$ as indicated by the static-copper measurements.

Components of elastic strain in the shock and transverse directions inferred from this linear fitting are listed in Table I together with the corresponding volumetric compression and the Hugoniot pressure and temperature obtained from EOS tables. ${ }^{40}$ Figure 10 shows these components of elastic strain, and the shear strain, as a function of pressure. The uncertainty of inferred strain $( \pm 0.002)$ corresponds to the uncertainty of these quantities that we found in fitting the data to the linear form of Eq. (1). We exclude from our later, detailed analysis of yield strength those measurements at the highest pressures $(>50 \mathrm{GPa})$ where increased width of the diffraction line and increased diffuse background signal on the BBXRD image plates result in the scatter or systematic error that makes accurate determination of the position of line centre difficult. But we note that for these few excluded cases, our VISAR data (Fig. 11) do nevertheless demonstrate near-constant freesurface velocity at late time (indicating a uniform state behind the shock front), and for completeness, we list the inferred components of strain for these cases also in Table I.

Figure 11 shows representative measurements of freesurface velocity obtained from VISAR, for experiments at 23, 42, and $68 \mathrm{GPa}$ pressure (respectively, Shots 5352, 4393, 


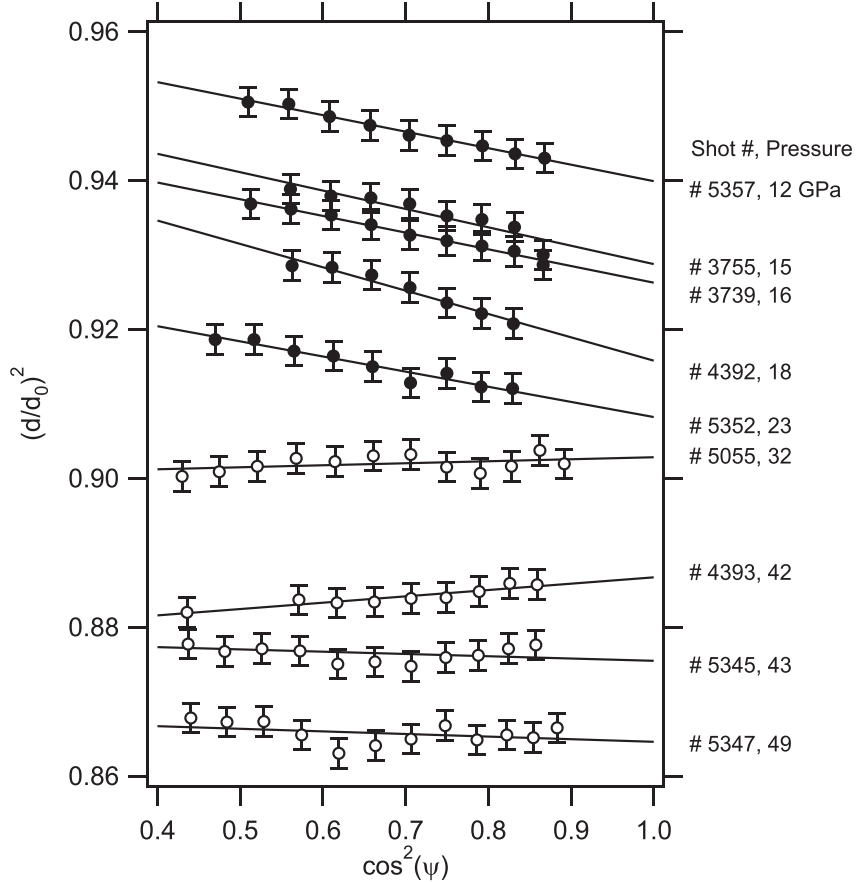

FIG. 9. Fitting of the measured (002) lattice-plane spacing of the compressed vanadium to the linear functional form proposed by Higginbotham and McGonegle. ${ }^{32} d$ and $d_{0}$ are the (002) lattice-plane spacings of the compressed and ambient materials, and $\psi$ is the angle between the shock direction (target normal) and the reciprocal-lattice vector of the compressed material. The data are labelled by shot number and pressure, and for each shot, the data points represent different azimuthal positions around the diffraction ring.

and 5350 of Table I). These measurements show a trend of smaller shock rise time at higher pressure, consistent with other shock experiments, ${ }^{27}$ and near-constant free-surface velocity at late time, indicating a near-uniform state behind the shock front. As we noted in Sec. II, the determination of fringe phase is subject to systematic uncertainty in a VISAR system of our type and is typically around \pm 0.05 fringe. The corresponding systematic uncertainty of free-surface velocity is of order $\pm 0.3 \mu \mathrm{m} \mathrm{ns}^{-1}$, and this is consistent with the differences of velocity recorded by the two VISAR channels, evident in Fig. 11. Our VISAR data encompass a field-ofview of 2-mm diameter at the sample and do reveal some transverse variation of surface velocity and shock breakout time that results from lateral non-uniformity of laser ablation pressure. Pressure inferred from free-surface velocity and pressure uncertainty inferred from transverse variations of free-surface velocity over the full 2-mm field-of-view of VISAR are listed in Table I. The free-surface-velocity data of Fig. 11 are mean values obtained from a region of the sample of the same width as that illuminated by the $\mathrm{x}$-ray probe beam used in the diffraction measurements. In those cases (not all experimental shots) where we obtained VISAR data, Fig. 12 compares our inferred equation-of-state (particle velocity $=0.5 \times$ free-surface velocity versus volumetric compression) with other published data.

In some cases (shots in the 4000 series), our VISAR measurements included a timing fiducial that enabled us to confirm that the x-ray diffraction measurement was made at a time when the shock front was close to a position half way
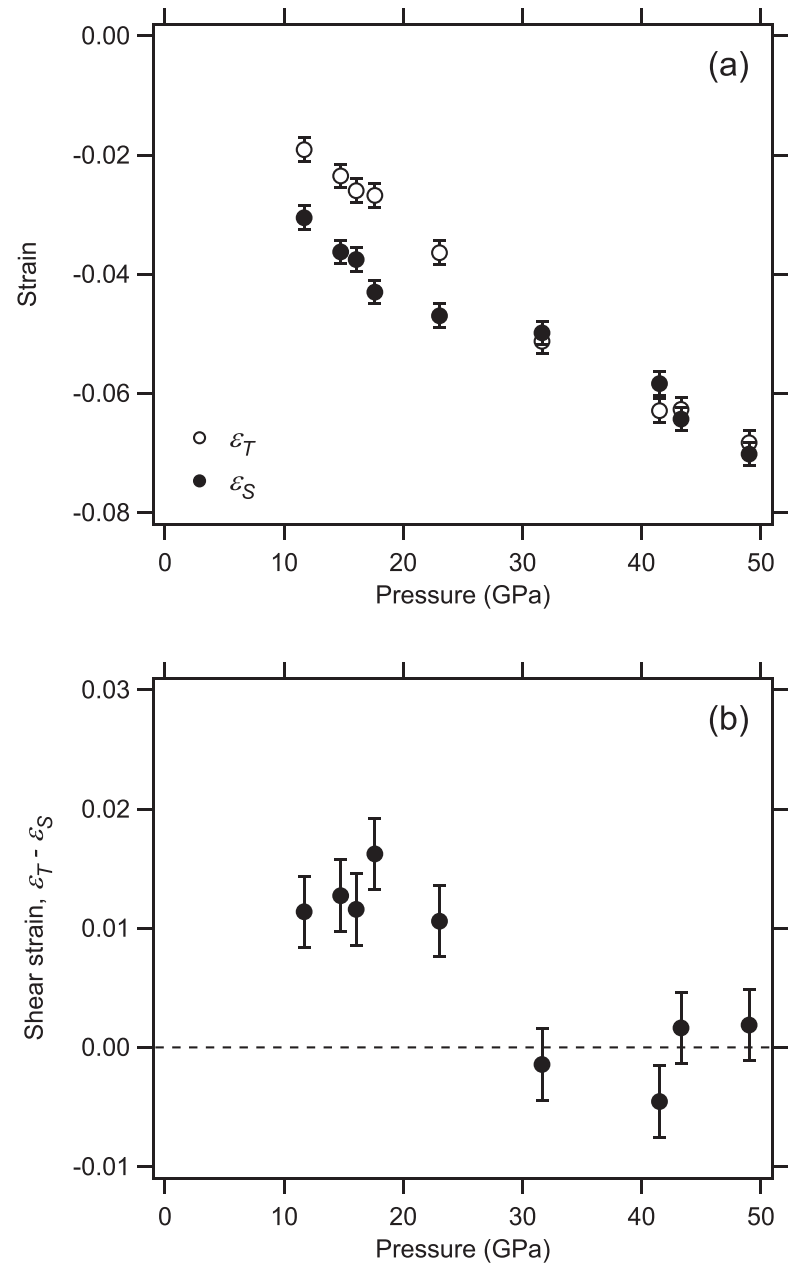

FIG. 10. (a) Normal (shock direction) and transverse components of volumeaveraged elastic strain and (b) shear strain inferred from experiment, as a function of drive pressure. Anisotropy of strain is not unambiguously identified for pressures above $30 \mathrm{GPa}$. The residual shear strain below $30 \mathrm{GPa}$ implies yield at a von Mises stress of order $1.5 \pm 0.3 \mathrm{GPa}$ (see Table I).

through the $10-\mu \mathrm{m}$ thickness vanadium sample. In other cases, when the timing fiducial was not available, achievement of this condition is in any case indicated by the comparable intensity of reflection in diffraction from the ambient and shocked material (Fig. 5).

\section{MODELLING}

Now, of course, our measurements of strain are volume averages, whereas strain in the shocked sample varies throughout its depth. Near the wavefront of the disturbance propagating through the material (where dislocation density is evolving rapidly), compression remains approximately uniaxial until the density of dislocations rises sufficiently for rapid stress relaxation and significant plastic flow to take place. Thereafter, strain remains more nearly constant throughout the compressed sample, with the degree of strain anisotropy, and the associated yield stress, set by the material state (dislocation density and related work hardening, temperature, and pressure) behind the shock front.

To provide insight into the distribution of elastic strain within the vanadium sample material, we have carried out 1-D simulations of the experiment using a hydrocode that 


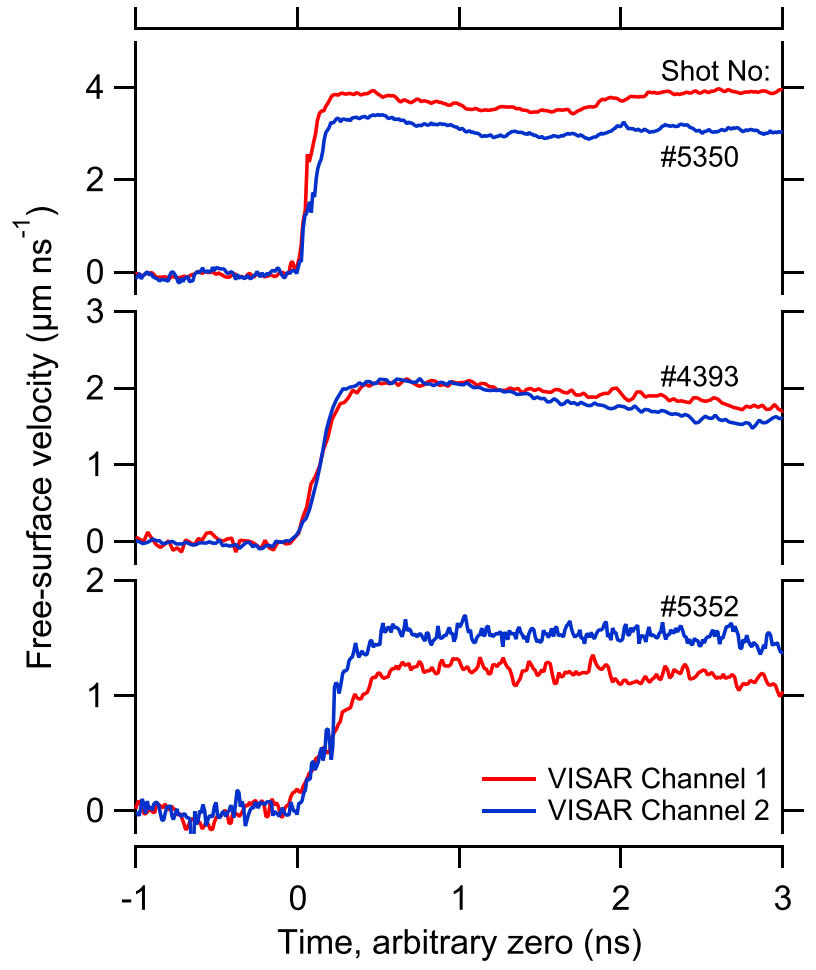

FIG. 11. VISAR measurements of free-surface velocity from representative examples of data covering the range of drive pressure $23 \mathrm{GPa}$ (Shot 5352), $42 \mathrm{GPa}$ (Shot 4393), and $68 \mathrm{GPa}$ (Shot 5350).

includes a treatment of material strength using an AWE dislocation-dynamics-based multi-scale crystal plasticity model, similar to that described in Ref. 26. This model is particularly appropriate because its treatment of plasticity accounts for the time-dependent evolution of dislocation density, to which the magnitude of residual strain anisotropy, and its distribution behind the shock front, is very sensitive. In the model, dislocation velocities in the thermal activation regime are described by an Arrhenius-type relation, while in the phonon drag regime, a temperature dependent linear drag

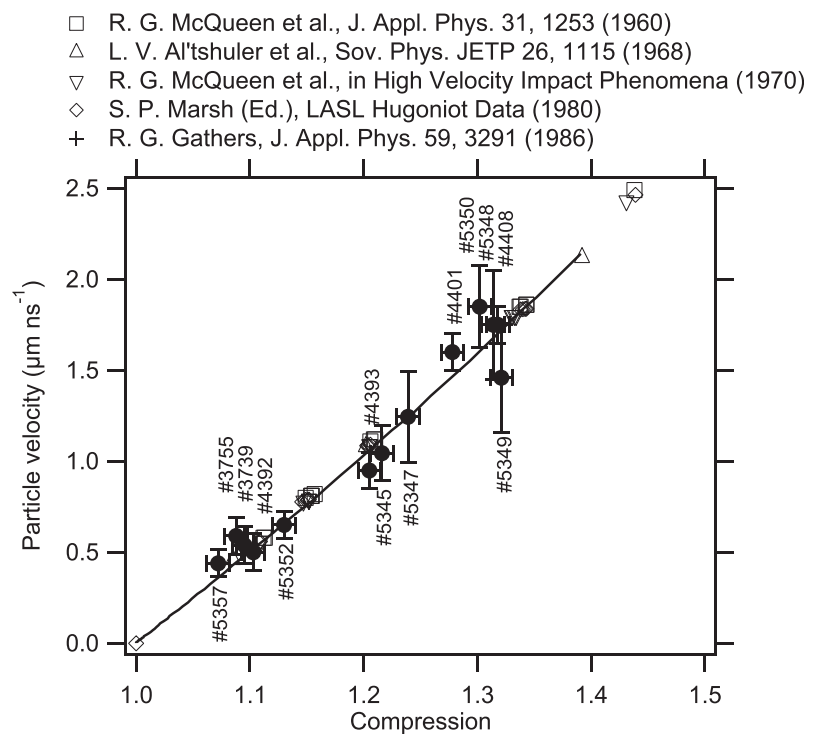

FIG. 12. Comparison of equation-of-state data inferred from this experiment (filled data points, identified by shot number) with other published data. relation is altered at high velocities to account for relativistic effects. Both mobile and immobile dislocation densities are evolved during deformation, with account taken of multiplication, annihilation, and immobilisation processes. Plasticity in the model depends on dislocation-mediated slip only. The dislocation-velocity and dislocation-density evolution relations are parameterised against MD calculations of dislocation mobility, and dislocation-dynamics calculations of saturation density, in vanadium reported in Ref. 23.

The simulations assume an initial dislocation density typical of a rolled polycrystalline metal of $5 \times 10^{13} \mathrm{~m}^{-2}$. The mobile dislocation-density multiplication rate was tuned to best match the free-surface-velocity data inferred from VISAR (Fig. 11) and was found to be consistent with that used in Ref. 23. Given the strong [100] texture exhibited in the experimental specimens, simulations assume single crystal vanadium oriented with the [100] direction normal to the surface of the sample, and having $10-\mu \mathrm{m}$ thickness. Stress is imposed instantaneously at one boundary of the material, driving a compression wave through its extent. Positiondependent components of elastic strain and dislocation density are extracted from the model, from which we generate synthetic X-ray diffraction data for comparison with experiment.

Figure 13 shows free-surface velocity data from simulations driven by surface-normal stresses of 23,42 , and $68 \mathrm{GPa}$ to match the experimental conditions of Fig. 11 (shot numbers 5352, 4393, and 5350, respectively). A clear Hugoniot elastic limit (HEL) is present in the simulations, indicating the transition from uniaxial elastic to plastic response, but is not evident in the experimental data. This difference may be related to three effects. First, the size of the VISAR spot used in the current experiments encompasses tens of

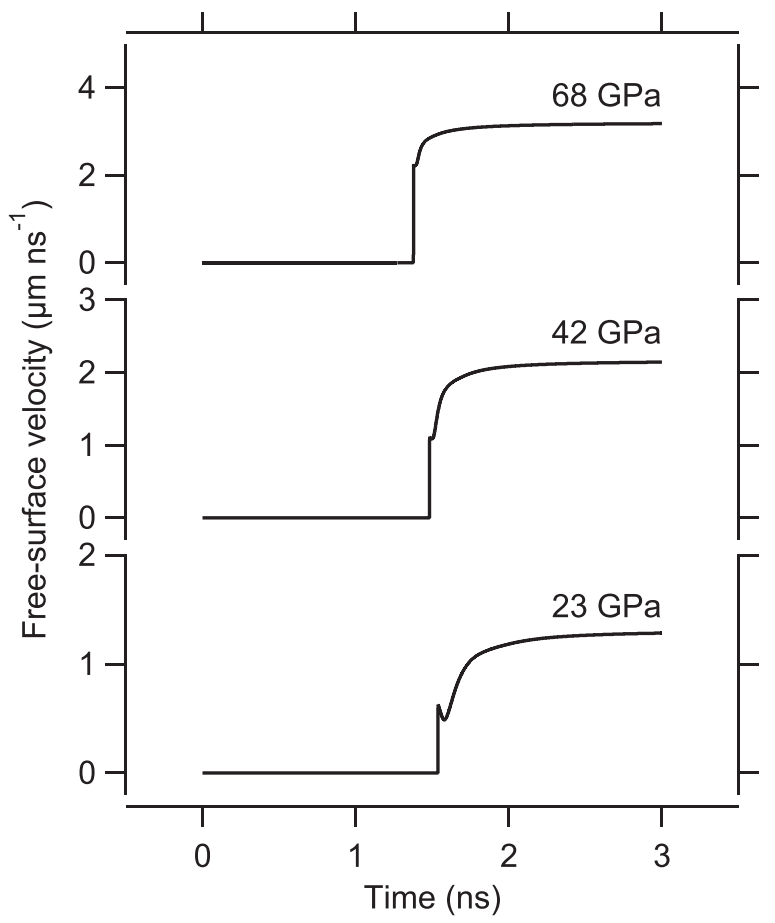

FIG. 13. Free-surface velocity from simulations of a $10-\mu \mathrm{m}$ thickness vanadium foil driven by 23,42 , and $68 \mathrm{GPa}$ normal stress and initial dislocation density of $5 \times 10^{13} \mathrm{~m}^{-2}$ (matching the experimental conditions of Fig. 10). 
thousands of grains. Lloyd et al. ${ }^{26}$ have shown that when averaging velocity profiles across 50 individual single crystal simulations, with crystal orientations varied to represent a fairly strong specimen texture, the average response of simulated laser shock experiments can change from a dual wave (in the single crystal case) to a single wave in the averaged case. Such a phenomenon could provide a more averaged "single-wave-like" observed response. Second, it is possible that some smearing of the wave front occurs when passing from grain to grain in the polycrystalline material, as described in Ref. 26. It is noted however that the shock wave has transmitted through only 1 to 2 grains at the time of diffraction in the current experiment, and so such smearing would be expected to be small. Third, the VISAR measurements recorded here may not be of sufficient resolution to distinguish the HEL features.

To match conditions representative of shot numbers 5357, 4392, 5055, and 5350, we have carried out simulations driven by $10,17,30$, and $68 \mathrm{GPa}$ surface-normal stresses. Figure 14 shows the components of elastic strain obtained from these simulations, as a function of position, and at a time when the wavefront of the disturbance has progressed approximately half-way through the material.

In generating synthetic x-ray diffraction data from the simulated strain, we take into account both the straindependent angle of peak reflection as well as the dislocationdensity-dependent diffraction line width.

We assume that line broadening arises predominantly from inhomogeneity of microscopic strain in each crystallite of the sample material. Bragg ${ }^{41}$ gives an approximate treatment of the diffraction line widths due to strain in which it is assumed that for a slip of magnitude $b$ and a crystallite dimension of $L$, the strain in the neighbourhood of a dislocation is of order $b / \mathrm{L}$. Since

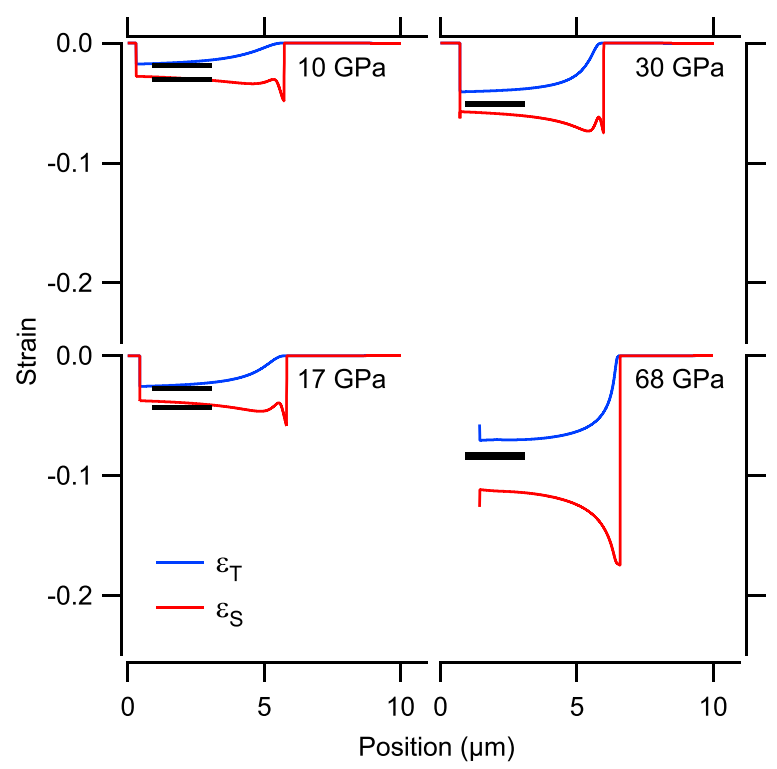

FIG. 14. Normal (shock direction) and transverse components of elastic strain from simulations of a $10-\mu \mathrm{m}$ thickness vanadium foil driven by 10 , 17,30 , and $68 \mathrm{GPa}$ normal stress and initial dislocation density of $5 \times 10^{13} \mathrm{~m}^{-2}$. The tick marks in black show the volume-averaged components of strain inferred from experiments under comparable conditions (shot numbers 5357, 4392, 5055, and 5350, respectively, of Table I).

$$
\Delta(\theta)=\frac{\Delta d}{d} \tan \theta
$$

it follows that line broadening due to inhomogeneity of strain throughout the crystallites is

$$
\Delta(2 \theta)_{\text {strain }}=2 \frac{b}{L} \tan \theta
$$

Now, with the magnitude of the Burgers vector $b$ equal to the lattice constant $a$ in the strained material, and for the (002) reflection that we have considered in making detailed measurements of diffraction line width, we have $b=a=2 d_{002}$. For dislocation density $\rho$, we have $L=(3 / \rho)^{1 / 2}$ (assuming the uniform threading in three dimensions of a unit cube by dislocation lines), and so for the (002) reflection

$$
\Delta(2 \theta)_{002, \text { strain }}=\frac{2}{\sqrt{3}} 2 d_{002} \frac{\sin \theta}{\cos \theta} \rho^{1 / 2}=1.15 \frac{\lambda}{\cos \theta} \rho^{1 / 2} .
$$

For each cell in the 1-D simulation, we generate a diffraction spectrum. We assume a monochromatic x-ray source of wavelength corresponding to the resonance line of heliumlike vanadium, and for each azimuthal position, $\varphi$, around the Debye-Scherrer ring (see Fig. 2), we calculate the diffraction angle of peak reflection, $2 \theta_{\mathrm{B}}$, resulting from the state of strain existing in that cell, and the diffraction line width resulting from the dislocation density in that cell. We represent diffraction by a Gaussian angular distribution of intensity with these properties of line-centre position and line width. By integration over the full extent of the sample (summation of a large number of such Gaussian contributions, from each cell of the simulation), we obtain synthetic x-ray diffraction data. These are shown in Fig. 15 and should be compared with the experimental data of Fig. 5.

We note in Fig. 15 that the region of near-uniaxial response of the sample results in a shoulder on the greaterangle side of the synthetic diffraction peak, and that this shoulder is most evident in simulations at the highest pressures where anisotropy of strain is greatest. This trend is not evident in the experimental data, and this may be a result of volumetric averaging by the $\mathrm{x}$-ray probe and the wave smearing discussed above (in the context of the lateral resolution of VISAR). Whereas at pressures below $30 \mathrm{GPa}$, simulation does reproduce the azimuthal dependence of diffraction angle recorded in the experiment, simulations at greater normal stress (30 and $70 \mathrm{GPa}$ ) do not: they show increased anisotropy of strain throughout the material, and a correspondingly greater dependence of diffraction angle on azimuthal angle. None of these features is evident in experiment. These differences are also apparent in Fig. 14, where the volumeaveraged strains inferred from experiment are shown by tick marks for comparison with the position-dependent strains found in simulation. At the lower pressures (10 and $17 \mathrm{GPa}$ ), the simulated strains in the region of plastic response are consistent with the experimental, volume-averaged measurement; at the higher pressures ( 30 and $68 \mathrm{GPa}$ ), this is not the case and no anisotropy of strain is evident in experiment. It is interesting to note in passing that the volumetric compression inferred from experiment at $\sim 30$ and $\sim 68 \mathrm{GPa}$ (Shots 5055 


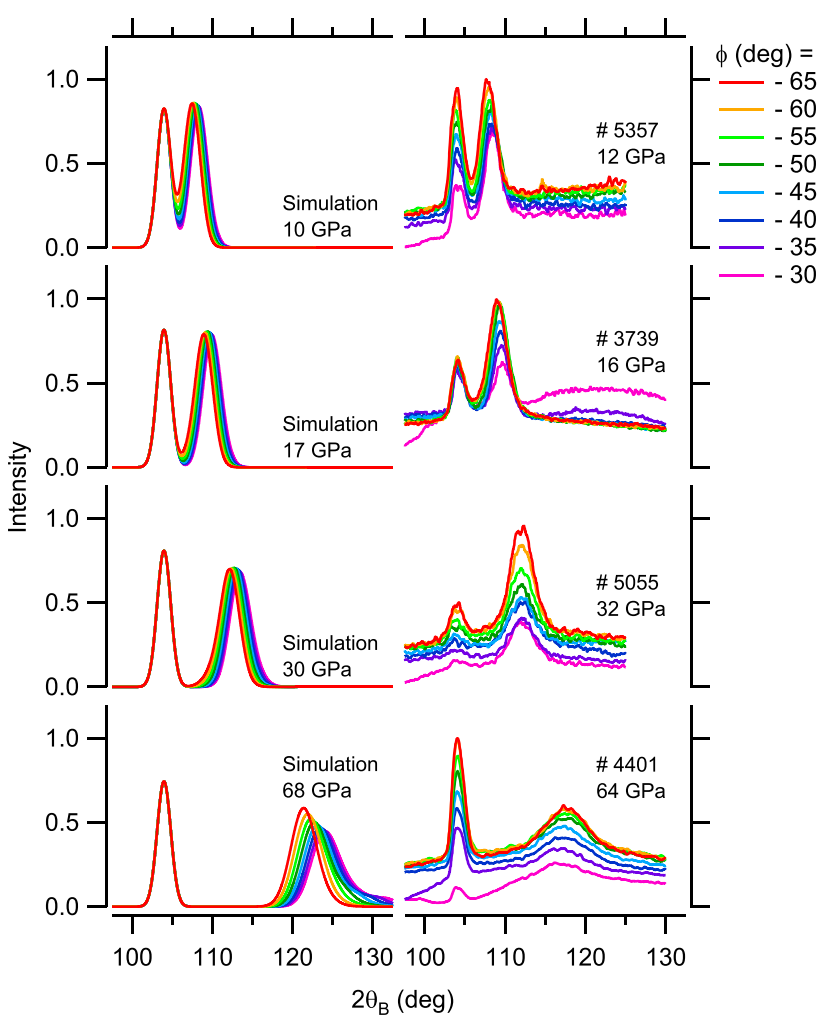

FIG. 15. Synthetic intensity distribution of x-ray diffraction, obtained by post-processing of the simulation data of Fig. 14 over the full extent of each simulation. The simulations (left-hand column) are compared with experimental data (right-hand column) at similar pressures. In the experimental data, the variation of intensity with position around the diffraction ring arises from the metallurgical texture of the vanadium sample, and this is not treated in the simulation.

and 5350) and volumetric compression evident in the region of plastic response in simulations at these pressures are similar.

It is interesting also to consider our measurements of the diffraction-line broadening. For consistency with our approach to the experimental data, we find the FWHM width of a Gaussian fit to the simulated x-ray diffraction data, at azimuthal angle $\varphi=-65^{\circ}$. This simulated diffraction line width is shown in Fig. 7. It is consistent with experimental data for the lower pressures accessed in the experiment, but not for the highest pressures (the four isolated data points, Shots 4401, 4408, 5348, and 5350 at 60-70 GPa).

Our measured strain data enable us to make a simple estimate of the limiting shear strength, or yield stress, of vanadium (under those conditions where we detect measurable anisotropy of strain). We assume a shear modulus, G, taken from the model by Steinberg and Guinan, ${ }^{20}$ and assume a limiting shear stress equal to the von Mises yield stress

$$
\bar{\sigma}=2 G\left(\varepsilon_{S}-\varepsilon_{T}\right) .
$$

Table I lists our assumed shear modulus and the resulting von Mises stress inferred from Eq. (2). We note again that for pressures $\geq 30 \mathrm{GPa}$, we find no unambiguously measurable anisotropy of strain (within the accuracy of the experiment) and therefore no clearly apparent limiting shear stress in the compressed vanadium. Figure 10 shows the measured strains, and the measured shear strain, as a function of pressure in the range of 10-50 GPa. In the region of 10-30 GPa, the von Mises yield stress is $1.2 \pm 0.3 \mathrm{GPa}$.

\section{DISCUSSION}

Our experimental data (volume-averaged components of strain inferred, within the limitations of the Voigt approximation, from in-situ x-ray diffraction measurements), and 1-D modelling using a dislocation-based multi-scale crystal plasticity strength model, are consistent up to the point where we infer a rather sudden disappearance of residual strain at shock pressures above $30 \mathrm{GPa}$. Above this pressure, modelling (based on dislocation-mediated plasticity alone) does not show the loss of strain anisotropy seen in the experimental data, although it is noted that model predictions in this high strain-rate, high pressure regime are expected to be inexact.

In contrast to the iso-strain Voigt approximation used in the analysis of our x-ray diffraction data, we could, in principle, have used the iso-stress Reuss approximation in the manner described in detail by MacDonald et al. ${ }^{42}$ However, this approach is complicated by the requirement for crystal elastic constants. Our sample material has a limited distribution of crystallite orientations, and in any case neither the Voigt nor Reuss approximations would be consistent with the disappearance of strain anisotropy that we find above $30 \mathrm{GPa}$.

Our data are susceptible, in principle, to compromise by the finite duration of the $\mathrm{x}$-ray pulse used to record the diffraction image, by a non-constant state behind the shock front (originating, for example, in temporal variations of laser ablation pressure, or the release that would arise from a late-time loss of ablation pressure), and from lateral variations of laser pressure (arising from transverse, spatial nonuniformity of laser illumination). In the context of loss of strain anisotropy, the $0.5 \mathrm{~ns}$ duration of the x-ray probe appears insignificant, and VISAR data (Fig. 11) indicate an adequately uniform state behind the shock front. Some transverse variation of shock breakout time and free-surface velocity was evident over the full 2-mm field of view of the VISAR diagnostic (as reported in the VISAR-inferred pressures listed in Table I), and this was most evident in some (but not all) shots at the highest shock pressures. However, we have found no correlation between these experiments with the greatest transverse non-uniformities of pressure and their corresponding $\mathrm{x}$-ray diffraction data (that in any case sample a smaller region of the shocked material than VISAR).

The loss of strain anisotropy may be the result of an increasingly complex microstructure in the region of plastic response, in which the multiplication of dislocations is succeeded at some critical shear stress by a fast relaxation of stress following the rapid formation of adiabatic shear bands (Nemat-Nasser and Guo ${ }^{43}$ ), twins, or the activation of homogeneous nucleation of dislocations. This would appear to be consistent with our strain-anisotropy data, and it is reminiscent of the rapid load drops observed in quasi-static tensile or compression tests in bcc metals following the nucleation of deformation twins, discussed by Christian and Mahajan ${ }^{39}$ 
and as seen in the continuum modelling of deformation twinning by, for example, Kochmann and Le. ${ }^{44}$ It finds a clear parallel in the MD simulations of tantalum single crystals shocked along the [001] direction by Higginbotham et al. ${ }^{12}$ and Tramontina et al., ${ }^{13}$ and we noted in Sec. III that in our experiment, the plane of maximum resolved shear stress in the vanadium-foil sample is close to the (112) twin composition planes for grain orientations with a high probability of occurrence. Such rapid stress relaxation may result in the deviatoric stress state accessed by our experiment no longer lying on the yield surface of the compressed material, so that the stress state we have diagnosed is not representative of the limiting yield stress. If so, this may explain differences between the pressure dependent yield-stress that we have found in our present work, and the experiments of Yu et al. ${ }^{17}$ which show a different trend of yield stress with shock pressure.

Clear evidence of twinning is expected at the positions identified in Fig. 3 in the $(2 \theta, \varphi)$ space of the diffraction images. At the highest shock pressures, we do indeed see the appearance of a diffraction feature in the (011) reflection close to $\varphi=0$, but it is of low intensity (almost lost in background) and evident only at the highest pressures we have accessed. We conclude that the current experiments do not show unequivocal evidence for twinning, and that twinning is therefore unlikely to be responsible for the observed loss of strength for shock pressures $>30 \mathrm{GPa}$.

It is expected that the homogeneous nucleation of dislocations ${ }^{45,46}$ would give rise to a large increase in dislocation population, and an associated increase in diffraction peak width, beyond that expected from dislocation multiplication. Indeed, as seen in Fig. 7, and discussed in Sec. V, measured peak widths are seen to increase at a faster rate with respect to shock pressure beyond the point at which strain anisotropy appears to be lost in the volume-averaged $\mathrm{x}$-ray diffraction data, in comparison to peak widths predicted by dislocation multiplication based modelling. These trends appear consistent with the initiation of homogeneous nucleation beyond the point at which strain anisotropy is lost. However, given uncertainties in modelling in this regime, and the lack of direct evidence in the current experiments, it is impossible to say, with confidence that such processes are indeed responsible.

In addition, the loss of strain anisotropy may be complicated by the presence of a phase transition, as suggested by the diamond-anvil-cell work of Jenei et al. ${ }^{19}$ in which the bcc-to-rhombohedral phase change appears to be evident under non-hydrostatic conditions at $30 \mathrm{GPa}$. Again, if the change of phase is rapid this could account for a rapid relaxation of stress and departure from the yield surface. However, we see no evidence for splitting of the cubic-basis (011) reflection into the expected closely spaced, rhombohedralbasis (011) and (001) reflections. We would expect a splitting of $2^{\circ}$ for a volumetric compression of $\geq 15 \%$, but this splitting might be masked by the line width. No splitting of the (002) reflection results from this phase change, by virtue of the crystallographic symmetry. A change of phase might result in the "annealing" of some lattice defects, and if so we would not expect the significantly greater diffraction line width for the (002) reflection that we see at the higher shock pressures. Conversely, in the event that crystallite size in the new phase is smaller than that in the parent material, a crystallite-size-related increase of line width might be expected.

Our multi-scale crystal plasticity strength model, which assumes single-phase bcc vanadium and dislocationmediated plasticity (without homogenous nucleation), does not show a loss of strain anisotropy above $30 \mathrm{GPa}$. An extended multi-scale continuum model that includes a description of the bcc-to-rhombohedral phase transformation has been reported by Barton in Ref. 47, but has not been employed here. Although under slightly different conditions, results in Ref. 47 suggest that this model shows a small decrease in yield stress when transforming to the rhombohedral phase at a strain-rate of $10^{7} \mathrm{~s}^{-1}$, albeit at a transformation pressure of $80 \mathrm{GPa}$, but no disappearance of the yield stress. Open questions remain in the interpretation of the loss of strain anisotropy in our present experiment, and therefore in the appropriate mechanisms to include in future developments of a model for material strength in this regime. The inclusion of twinning, homogeneous dislocation nucleation, phase change, and the effects of deviatoric stress on phase transformation, to name but a few, would be interesting extensions to explore in future modelling studies.

In summary, we have reported experiments in which powder-diffraction data were recorded from polycrystalline vanadium foils, shock-compressed to pressures in the range of $10-70 \mathrm{GPa}$. We find residual anisotropic strain corresponding to yield strength of $1.2 \mathrm{GPa}-1.8 \mathrm{GPa}$ for shock pressures below $30 \mathrm{GPa}$, but significantly less anisotropy of strain in the range of shock pressures above this. The current diffraction experiments do not provide sufficient evidence to unequivocally explain the latter observation, although possible contributing mechanisms are discussed.

\section{ACKNOWLEDGMENTS}

We are grateful for the skilled and willing support of all the target-area and laser staff of the Orion facility and the AWE target-fabrication group. J.S.W. is grateful for support from EPSRC under Grant No. EP/J017256/1.

${ }^{1}$ B. J. Jensen and Y. M. Gupta, J. Appl. Phys. 104, 013510 (2008).

${ }^{2}$ B. A. Remington, R. E. Rudd, and J. S. Wark, Phys. Plasmas 22, 090501 (2015).

${ }^{3}$ T. P. Remington, B. A. Remington, E. N. Hahn, and M. A. Meyers, Mater. Sci. Eng., A 688, 429 (2017).

${ }^{4}$ R. E. Rudd, A. Arsenlis, N. R. Barton, R. M. Cavallo, A. J. Comley, B. R. Maddox, J. Marian, H.-S. Park, S. T. Prisbey, C. E. Wehrenberg, L. Zepeda-Ruiz, and B. A. Remington, J. Phys.: Conf. Ser. 500, 112055 (2014).

51. Dubrovinsky, N. Dubrovinskaia, V. B. Prakapenka, and A. M. Abakumov, Nat. Commun. 3, 1163 (2012).

${ }^{6}$ W. J. Murphy, A. Higginbotham, G. Kimminau, B. Barbrel, E. M. Bringa, J. Hawreliak, R. Kodama, M. Koenig, W. McBarron, M. A. Meyers, B. Nagler, N. Ozaki, N. Park, B. Remington, S. Rothman, S. M. Vinko, T. Whitcher, and J. S. Wark, J. Phys.: Condens. Matter 22, 065404 (2010).

${ }^{7}$ D. H. Kalantar, J. F. Belak, G. W. Collins, J. D. Colvin, H. M. Davies, J. H. Eggert, T. C. Germann, J. Hawreliak, B. L. Holian, K. Kadau, P. S. Lomdahl, H. E. Lorenzana, M. A. Meyers, K. Rosolankova, M. S. Scheinder, J. Sheppard, J. S. Stölken, and J. S. Wark, Phys. Rev. Lett. 95, 075502 (2005).

${ }^{8}$ J. Hawreliak, J. D. Colvin, J. H. Eggert, D. H. Kalantar, H. E. Lorenzana, J. S. Stölken, H. M. Davies, T. C. Germann, B. L. Holian, K. Kadau, P. S. Lomdahl, A. Higginbotham, K. Rosolankova, J. Sheppard, and J. S. Wark, Phys. Rev. B 74, 184107 (2006). 
${ }^{9}$ J. A. Hawreliak, B. El-Dasher, H. Lorenzana, G. Kimminau, A. Higginbotham, B. Nagler, S. M. Vinko, W. J. Murphy, T. Whitcher, J. S. Wark, S. Rohman, and N. Park, Phys. Rev. B 83, 144114 (2011).

${ }^{10}$ A. J. Comley, B. R. Maddox, R. E. Rudd, S. T. Prisbey, J. A. Hawrelaik, D. A. Orlikowski, S. C. Peterson, J. H. Satcher, A. J. Elsholz, H.-S. Park, B. A. Remington, N. Bazin, J. M. Foster, P. Graham, N. Park, P. A. Rosen, S. R. Rothman, A. Higginboham, M. Suggit, and J. S. Wark, Phys. Rev. Lett. 110, 115501 (2013).

${ }^{11}$ C. E. Wehrenberg, A. J. Comley, N. R. Barton, F. Coppari, D. Fratanduono, C. M. Huntington, B. R. Maddox, H.-S. Park, C. Plechaty, S. T. Prisbey, B. A. Remington, and R. E. Rudd, Phys. Rev. B 92, 104305 (2015).

${ }^{12}$ A. Higginbotham, M. J. Suggit, E. M. Bringa, P. Erhart, J. A. Hawreliak, G. Mogni, N. Park, B. A. Remington, and J. S. Wark, Phys. Rev. B 88, 104015 (2013).

${ }^{13}$ D. Tramontina, P. Erhart, T. Germann, J. Hawreliak, A. Higginbotham, N. Park, R. Ravelo, A. Stukowski, M. Suggit, Y. Tang, J. Wark, and E. Bringa, High Energy Density Phys. 10, 9 (2014).

${ }^{14}$ G. Kimminau, B. Nagler, A. Higginbotham, W. J. Murphy, N. Park, J. Hawreliak, K. Kadau, T. C. Germann, E. M. Bringa, D. H. Kalantar, H. E. Lorenzana, B. A. Remington, and J. S. Wark, J. Phys.: Condens. Matter 20, 505203 (2008).

${ }^{15}$ L. C. Chhabildas and C. R. Hills, in Metallurgical Applications of ShockWave and High-Strain-Rate Phenomena, edited by L. E. Murr, K. P. Staudhammer, and M. A. Meyers (Marcel Dekker Publisher, New York, 1986), pp. 429-488.

${ }^{16}$ H.-S. Park, B. A. Remington, R. C. Becker, J. V. Bernier, R. M. Cavallo, K. T. Lorenz, S. M. Pollaine, S. T. Prisbey, R. E. Rudd, and N. R. Barton, Phys. Plasmas 17, 056314 (2010).

${ }^{17}$ Y. Yu, Y. Tan, C. Dai, X. Li, Y. Li, Q. Wu, and H. Tan, Appl. Phys. Lett. 105, 201910 (2014)

${ }^{18}$ J.-H. P. Klepeis, H. Cynn, W. J. Evans, R. E. Rudd, L. H. Yang, H. P. Liermann, and W. Yang, Phys. Rev. B 81, 134107 (2010).

${ }^{19}$ Z. Jenei, H. P. Liermann, H. Cynn, J.-H. P. Klepeis, B. J. Baer, and W. J. Evans, Phys. Rev. B 83, 054101 (2011).

${ }^{20}$ D. J. Steinberg, S. G. Cochran, and M. W. Guinan, J. Appl. Phys. 51, 1498 (1980).

${ }^{21}$ D. J. Steinberg and C. M. Lund, J. Appl. Phys. 65, 1528 (1989).

${ }^{22}$ D. L. Preston, D. L. Tonks, and D. C. Wallace, J. Appl. Phys. 93, 211 (2003).

${ }^{23}$ N. R. Barton, J. V. Bernier, R. Becker, A. Arsenlis, R. Cavallo, J. Marian, M. Rhee, H.-S. Park, B. A. Remington, and R. T. Olson, J. Appl. Phys. 109, 073501 (2011).

${ }^{24}$ B. L. Hansen, I. J. Beyerlein, C. A. Bronkhorst, E. K. Cerreta, and D. Dennis-Koller, Int. J. Plast. 44, 129 (2013).

${ }^{25}$ J. L. Ding and J. R. Asay, J. Appl. Phys. 109, 083505 (2011).
${ }^{26}$ J. T. Lloyd, J. D. Clayton, R. Becker, and D. L. McDowell, Int. J. Plast. 60, 118 (2014).

${ }^{27}$ J. W. Swegle and D. E. Grady, J. Appl. Phys. 58, 692 (1985).

${ }^{28}$ C. H. Lu, B. A. Remington, B. R. Maddox, B. Kad, H. S. park, S. T. Prisbrey, and M. A. Meyers, Acta. Mater. 60, 6601 (2012).

${ }^{29}$ J. N. Florando, N. R. Barton, B. S. El-Dasher, J. M. McNaney, and M. Kumar, J. Appl. Phys. 113, 083522 (2013).

${ }^{30}$ K. Kadau, T. C. Germann, P. S. Lomdahl, and B. L. Holian, Science 296, 1681 (2002)

${ }^{31}$ N. Hopps, K. Oades, J. Andrew, C. Brown, G. Cooper, C. Danson, S. Daykin, S. Duffield, R. Edwards, D. Egan, S. Elsmere, S. Gales, M. Girling, E. Gumbrell, E. Harvey, D. Hillier, D. Hoarty, C. Horsfield, S. James, A. Leatherland, S. Masoero, A. Meadowcroft, M. Norman, S. Parker, S. Rothman, M. Rubery, P. Treadwell, D. Winter, and T. Bett, Plasma Phys. Controlled Fusion 57, 064002 (2015).

${ }^{32}$ A. Higginbotham, P. G. Stubley, A. J. Comley, J. H. Eggert, J. M. Foster, D. H. Kalantar, D. McGonegle, S. Patel, L. J. Peacock, S. D. Rothman, R. F. Smith, M. J. Suggit, and J. S. Wark, Sci. Rep. 6, 24211 (2016).

${ }^{33}$ Goodfellow Cambridge Ltd., Ermine Business Park, Huntingdon, PE29 6WR, U.K.

${ }^{34}$ We are grateful to Jim Lynn, Department of Physics, University of Oxford for careful metrology of the BBXRD camera.

${ }^{35}$ P. M. Celliers, D. K. Bradley, G. W. Collins, D. G. Hicks, T. R. Boehly, and W. J. Armstrong, Rev. Sci. Instrum. 75, 4916 (2004).

${ }^{36}$ EBSD measurements were made using a JEOL JSM7000F scanning electron microscope, JEOL USA, 11 Dearborn Road, Peabody, MA 01960, USA.

${ }^{37}$ A. Higginbotham and D. McGonegle, J. Appl. Phys. 115, 174906 (2014).

${ }^{38}$ J. S. Kallend, in Texture and Anisotropy, edited by U. F. Kocks, C. N. Tomé, and H.-R. Wenk (Cambridge University Press, 1998), pp. 102-124.

${ }^{39}$ J. W. Christian and S. Mahajan, Prog. Mater. Sci. 39, 1 (1995).

${ }^{40}$ K. S. Holian, "SESAME tables," Los Alamos National Laboratory Report No. LA-UR-92-3407, 1992.

${ }^{41}$ W. L. Bragg, Proc. Cambridge Philos. Soc. 45, 125 (1949).

${ }^{42}$ M. J. MacDonald, J. Vorberger, E. J. Gamboa, R. P. Drake, S. H. Glenzer, and L. B. Fletcher, J. Appl. Phys. 119, 215902 (2016).

${ }^{43}$ S. Nemat-Nasser and W. Guo, Mech. Mater. 32, 243 (2000).

${ }^{44}$ D. M. Kochmann and K. C. Le, J. Mech. Phys. Solids 57, 987 (2009).

${ }^{45}$ R. E. Rudd, A. J. Comley, J. Hawreliak, B. R. Maddox, H.-S. Park, and B. A. Remington, AIP Conf. Proc. 1426, 1379-1382 (2012).

${ }^{46}$ E. M. Bringa, K. Rololankova, R. E. Rudd, B. A. Remington, J. S. Wark, M. Duchaineau, D. H. Kalantar, J. Hawreliak, and J. Belak, Nat. Mater. 5, 805-809 (2006).

${ }^{47}$ N. R. Barton, A. Arsenlis, M. Rhee, J. Marian, J. V. Bernier, M. Tang, and L. Yangyang, "A multi-scale strength model with phase transformation," AIP Conf. Proc. 1426, 1513-1516 (2012). 